\title{
A Compact Multi-planet System with a Significantly Misaligned Ultra Short Period Planet
}

Rodriguez, Joseph E.; Becker, Juliette C.; Eastman, Jason D.; Hadden, Sam; Vanderburg, Andrew; Khain, Tali; Quinn, Samuel N.; Mayo, Andrew; Dressing, Courtney D.; Schlieder, Joshua E. Total number of authors:

23

Published in:

Astronomical Journal

Link to article, DOI:

10.3847/1538-3881/aae530

Publication date:

2018

Document Version

Publisher's PDF, also known as Version of record

Link back to DTU Orbit

Citation (APA):

Rodriguez, J. E., Becker, J. C., Eastman, J. D., Hadden, S., Vanderburg, A., Khain, T., Quinn, S. N., Mayo, A., Dressing, C. D., Schlieder, J. Ė., Ciardi, D. R., Latham, D. W., Rappaport, S., Adams, F. C., Berlind, P., Bieryla, A., Calkins, M. L., Esquerdo, G. A., Kristiansen, M. H., ... Terentev, I. (2018). A Compact Multi-planet System with a Significantly Misaligned Ultra Short Period Planet. Astronomical Journal, 156(5), [245]. https://doi.org/10.3847/1538-3881/aae530

\section{General rights}

Copyright and moral rights for the publications made accessible in the public portal are retained by the authors and/or other copyright owners and it is a condition of accessing publications that users recognise and abide by the legal requirements associated with these rights.

- Users may download and print one copy of any publication from the public portal for the purpose of private study or research.

- You may not further distribute the material or use it for any profit-making activity or commercial gain

- You may freely distribute the URL identifying the publication in the public portal 


\title{
A Compact Multi-planet System with a Significantly Misaligned Ultra Short Period Planet
}

\author{
Joseph E. Rodriguez ${ }^{1}$ (D), Juliette C. Becker ${ }^{2}$ (i), Jason D. Eastman ${ }^{1}$ (i), Sam Hadden ${ }^{1}$, Andrew Vanderburg ${ }^{3,15}$ (D), Tali Khain ${ }^{4}$ (i), \\ Samuel N. Quinn ${ }^{1}(\mathbb{D})$, Andrew Mayo ${ }^{5,6,7,16,17}$, Courtney D. Dressing ${ }^{7}$ (D) , Joshua E. Schlieder ${ }^{8}$ (iD, David R. Ciardi ${ }^{9}$,

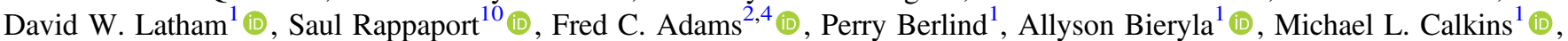 \\ Gilbert A. Esquerdo $^{1}$, Martti H. Kristiansen ${ }^{5,11}$, Mark Omohundro ${ }^{12}$, Hans Martin Schwengeler ${ }^{12}$, Keivan G. Stassun ${ }^{13,14}$ (1) , and \\ Ivan Terentev ${ }^{12}$ \\ ${ }^{1}$ Harvard-Smithsonian Center for Astrophysics, 60 Garden St, Cambridge, MA 02138, USA \\ 2 Astronomy Department, University of Michigan, 1085 S University Avenue, Ann Arbor, MI 48109, USA \\ ${ }^{3}$ Department of Astronomy, The University of Texas at Austin, Austin, TX 78712, USA \\ ${ }^{4}$ Physics Department, University of Michigan, Ann Arbor, MI 48109, USA \\ ${ }^{5}$ DTU Space, National Space Institute, Technical University of Denmark, Elektrovej 327, DK-2800 Lyngby, Denmark \\ ${ }^{6}$ Centre for Star and Planet Formation, Niels Bohr Institute \& Natural History Museum, University of Copenhagen, DK-1350 Copenhagen, Denmark \\ ${ }_{7}$ Astronomy Department, University of California Berkeley, Berkeley, CA 94720-3411, USA \\ ${ }^{8}$ Exoplanets \& Stellar Astrophysics Laboratory, Code 667, NASA Goddard Space Flight Center, Greenbelt, MD, USA \\ ${ }^{9}$ NASA Exoplanet Science Institute, California Institute of Technology, Pasadena, CA, USA
${ }^{10}$ Department and Kavli Institute for Astrophysics and Space Research, Massachusetts Institute of Technology, Cambridge, MA 02139, USA \\ ${ }^{11}$ Brorfelde Observatory, Observator Gyldenkernes Vej 7, DK-4340 Tølløse, Denmark \\ ${ }^{12}$ Citizen Scientist \\ ${ }^{13}$ Department of Physics and Astronomy, Vanderbilt University, Nashville, TN 37235, USA \\ ${ }^{14}$ Department of Physics, Fisk University, Nashville, TN 37208, USA \\ Received 2018 June 21; revised 2018 September 26; accepted 2018 September 28; published 2018 November 2
}

\begin{abstract}
We report the discovery of a compact multi-planet system orbiting the relatively nearby (78 pc) and bright $(K=8.9)$ K-star, K2-266 (EPIC 248435473). We identify up to six possible planets orbiting K2-266 with estimated periods of $P_{b}=0.66, P_{.02}=6.1, P_{c}=7.8, P_{d}=14.7, P_{e}=19.5$, and $P_{.06}=56.7$ days, and radii of $R_{P}=3.3 R_{\oplus}, 0.646 R_{\oplus}$, $0.705 R_{\oplus}, 2.93 R_{\oplus}, 2.73 R_{\oplus}$, and $0.90 R_{\oplus}$, respectively. We are able to confirm the planetary nature of two of these planets ( $\mathrm{d}$ and e) by analyzing their transit timing variations $\left(m_{d}=8.9_{-3.8}^{+5.7} M_{\oplus}\right.$ and $\left.m_{e}=14.3_{-5.0}^{+6.4} M_{\oplus}\right)$, confidently validate the planetary nature of two other planets (b and c), and classify the last two as planetary candidates (K2-266.02 and .06). From a simultaneous fit of all six possible planets, we find that K2-266 b’s orbit has an inclination of $75^{\circ} .32$ while the other five planets have inclinations of $87^{\circ}-90^{\circ}$. This observed mutual misalignment may indicate that $\mathrm{K} 2-266 \mathrm{~b}$ formed differently from the other planets in the system. The brightness of the host star and the relatively large size of the sub-Neptune sized planets $d$ and e make them well-suited for atmospheric characterization efforts with facilities like the Hubble Space Telescope and upcoming James Webb Space Telescope. We also identify an 8.5 day transiting planet candidate orbiting EPIC 248435395, a co-moving companion to K2-266.
\end{abstract}

Key words: planetary systems - planets and satellites: detection - stars: individual (K2-266, EPIC 248435395)

\section{Introduction}

Our understanding of exoplanet demographics has rapidly expanded as a direct result of the success of the Kepler and K2 missions. With the successful launch of the Transiting Exoplanet Survey Satellite mission, which is expected to discover thousands of new exoplanetary systems (Ricker et al. 2015), the community is now focused on understanding the mechanisms responsible for the diversity of exoplanet architectures. We now know of over 700 multi-planet systems and a total of more than 3700 confirmed or validated planets to date. ${ }^{18}$ From these discoveries, we know that the most commonly known planets with periods $P<100$ days are smaller than Neptune, a large fraction of which are super-Earths and mini-Neptunes $\left(R_{P}=1.5-4 R_{\oplus}\right.$; Fressin et al. 2013). With no analogues in our own solar system, our understanding of these planets is limited.

\footnotetext{
15 NASA Sagan Fellow.

${ }^{16}$ National Science Foundation Graduate Research Fellow.

${ }^{17}$ Fulbright Fellow.

18 https://exoplanetarchive.ipac.caltech.edu/
}

The large number of multi-planet systems discovered may provide key information to facilitate our understanding of the formation of our own solar system. From studying multi-planet systems using Kepler data, it has been determined that $\sim 30 \%$ of Sun-like stars have compact and closely aligned architectures, with planetary radii $R_{P}>R_{\oplus}$ and orbital periods less than 400 days (Zhu et al. 2018). Planets in systems with large mutual inclinations, however, might not all transit. The mutual inclination could be dependent on the number of planets in the system. Specifically, systems with fewer planets have larger mutual inclinations, possibly explaining the observed excess of Kepler single planet systems (Zhu et al. 2018). If unaccounted for, this bias can affect our understanding of planet formation. Fortunately, typical mutual inclinations within transiting systems can be constrained by measuring the ratio of transit durations of adjacent transiting planets. Studies that constrain the underlying multiplicity and distribution of inclinations suggest that transiting multi-planet systems are close to aligned, with mutual inclinations typically less than a few degrees (Fang \& Margot 2012; Figueira et al. 2012; Swift et al. 2013; Fabrycky et al. 2014; Ballard \& Johnson 2016). However, 
many studies have shown that the observed population is not well-represented by a single-component model (Lissauer et al. 2011; Ballard \& Johnson 2016), and this claim is supported by simulations of late-stage planet formation (Hansen \& Murray 2013); the underlying population may consist of some well-aligned systems and some with large mutual inclinations.

Ultra short period (USP) planets, planets that orbit with periods less than a day, may provide insight into the origin of mutually misaligned planetary systems. These are relatively rare objects ( $0.5 \%$ of all stars) (Sanchis-Ojeda et al. 2014), but their close proximity to their host star allows them to transit at a larger range of inclinations relative to our line of sight. This relatively high transit probability makes the USP planet in a multi-planet system more likely to transit than the longerperiod planets in the system (e.g., 55 Cancri) (Fischer et al. 2008; Batalha et al. 2011). It also makes it more likely that misaligned systems containing USP planets will be observed to host multiple transiting planets, which affords greater opportunities for detailed investigations of the physical and dynamical properties of the planets. USP planets therefore have the potential to help us understand the origin of planetary systems with high mutual inclinations.

Because young stars are larger in radius than their sizes on the main sequence, by factors of 3-4, it is unlikely that USP planets could form in situ: the host star would have engulfed some of the known USP planets, based on stellar properties derived from pre-main-sequence evolutionary tracks (Palla \& Stahler 1991; D’Antona \& Mazzitelli 1994). As a result, one possible origin scenario is that USP planet migration is influenced by gravitational interactions with other planets or stars, increasing the planet's orbital eccentricity. This "High Eccentricity Migration" mechanism (HEM), has also been proposed to explain the origin of hot Jupiters (e.g., Petrovich et al. 2018). These systems initially retain their primordial eccentricities from these interactions (Rasio \& Ford 1996; Wu \& Murray 2003; Fabrycky \& Tremaine 2007; Nagasawa \& Ida 2011; Wu \& Lithwick 2011), but subsequent tidal interactions should circularize the orbit (e.g., Adams \& Laughlin 2006). However, the inclination excited by HEM may remain, resulting in highly misaligned planetary orbits.

Another possible explanation for misaligned planetary systems is that they originate from misaligned disks around young stars. It is known that young stars are surrounded by circumstellar disks of gas and dust that eventually form the planetary systems that are observed today. From highresolution observations of these circumstellar disks-for example, using the Atacama Large Millimeter/submillimeter Array (ALMA) - we know that these disks are not smooth and uniform. Instead they contain gaps or rings (ALMA Partnership et al. 2015), and display misalignment with their disks and even multiple disks (e.g., Beta Pic) (Heap et al. 2000). Additionally, wide binary systems where each star has its own circumstellar disk have been shown to be mutually misaligned (e.g., Jensen \& Akeson 2014 and references therein).

Using high-precision photometric observations from Spitzer and $K 2$, a sub-class of young stellar objects called "dippers" have been identified; they display large-amplitude $(>10 \%)$ dimming events that occur on timescales of days (Alencar et al. 2010; Morales-Calderón et al. 2011; Cody et al. 2014; Ansdell et al. 2016b). The observed variability has been attributed to extinction by dust in the inner disk, implying that disks would need to be relatively edge-on, as suggested for the archetypal dipper AA Tau (Bouvier et al. 1999). However, recent high spatial resolution millimeter mapping of AA Tau by ALMA shows a modestly inclined disk at $59^{\circ} .1$ (Loomis et al. 2017). Even more extreme examples exist, such as the dipper J1604-2130, for which ALMA observations reveal the disk to be nearly face-on (Ansdell et al. 2016a). These observations, combined with the observed photometric dimming events, suggest that the inner disk is more aligned to our line of sight-and therefore misaligned relative to the outer disk. Finally, we note that molecular cloud cores that form stars do not have perfectly well-ordered distributions of angular momentum, so the formation of disks, and later planets, naturally produces some misalignment (e.g., Spalding et al. 2014 and references therein).

Multi-planet systems also allow us to determine key physical planet parameters, such as mass and orbital eccentricity, through the detection and analysis of transit timing and duration variations (TTV and TDV, respectively) (Agol et al. 2005; Holman \& Murray 2005). The slight variations in the timing between consecutive transits are caused by another planet in the system, and result from exchanging energy and angular momentum due to their mutual gravitational interaction. Systems that have planets in or near mean motion resonance (MMR) can produce large amplitude timing variations, allowing the measurement of mass and eccentricity for small planets with longer periods. Efforts to analyze the TTVs for a large sample of planetary systems have provided mass and eccentricity measurements for planets that would not be accessible from other techniques, such as radial velocities (Steffen et al. 2013; Holczer et al. 2016; Jontof-Hutter et al. 2016; Hadden \& Lithwick 2017).

In this paper, we present the discovery and characterization of a compact multi-planet system orbiting the late K-star K2-266 (EPIC 248435473; see Table 1). Using observations from the $K 2$ mission, we have identified up to six planets orbiting K2-266, with periods of $0.66,6.1,7.8,14.7,19.5$, and 56.7 days. We are able to confidently confirm the planetary nature of two of these planets $\left(P_{d}=14.7\right.$ days and $P_{e}=$ 19.5 days $)$, validate two more as planets $\left(P_{b}=0.66\right.$ days and $P_{c}=7.8$ days), and we classify the other two (weaker) signals as planetary candidates. From a simultaneous global model of all six planets and candidates, we find that the orbit of K2-266 b has an inclination of $75^{\circ} .32$, while the other five planets and candidates have inclinations of $87^{\circ}$ to $90^{\circ}$. This significant misalignment of the inner planet has interesting implications for the dynamical history of the system, and may suggest that it had a different evolutionary path than the rest of the planets. Additionally, K2-266 has a co-moving companion, EPIC 248435395, that is $42^{\prime \prime}$ away and an early M-star. This companion was resolved by $K 2$, and we report the identification of a planet candidate orbiting EPIC 248435395 with a period of 8.5 days.

The paper is organized in the following way: We first discuss our photometric and spectroscopic observations in Section 2. Our EXOFASTv2 global model methodology and results are then presented in Section 3. We present all observations on the co-moving companion (EPIC 248435395) and discuss the nature of the companion star and its planetary candidate in Section 5. A dynamical analysis of the system is carried out in 


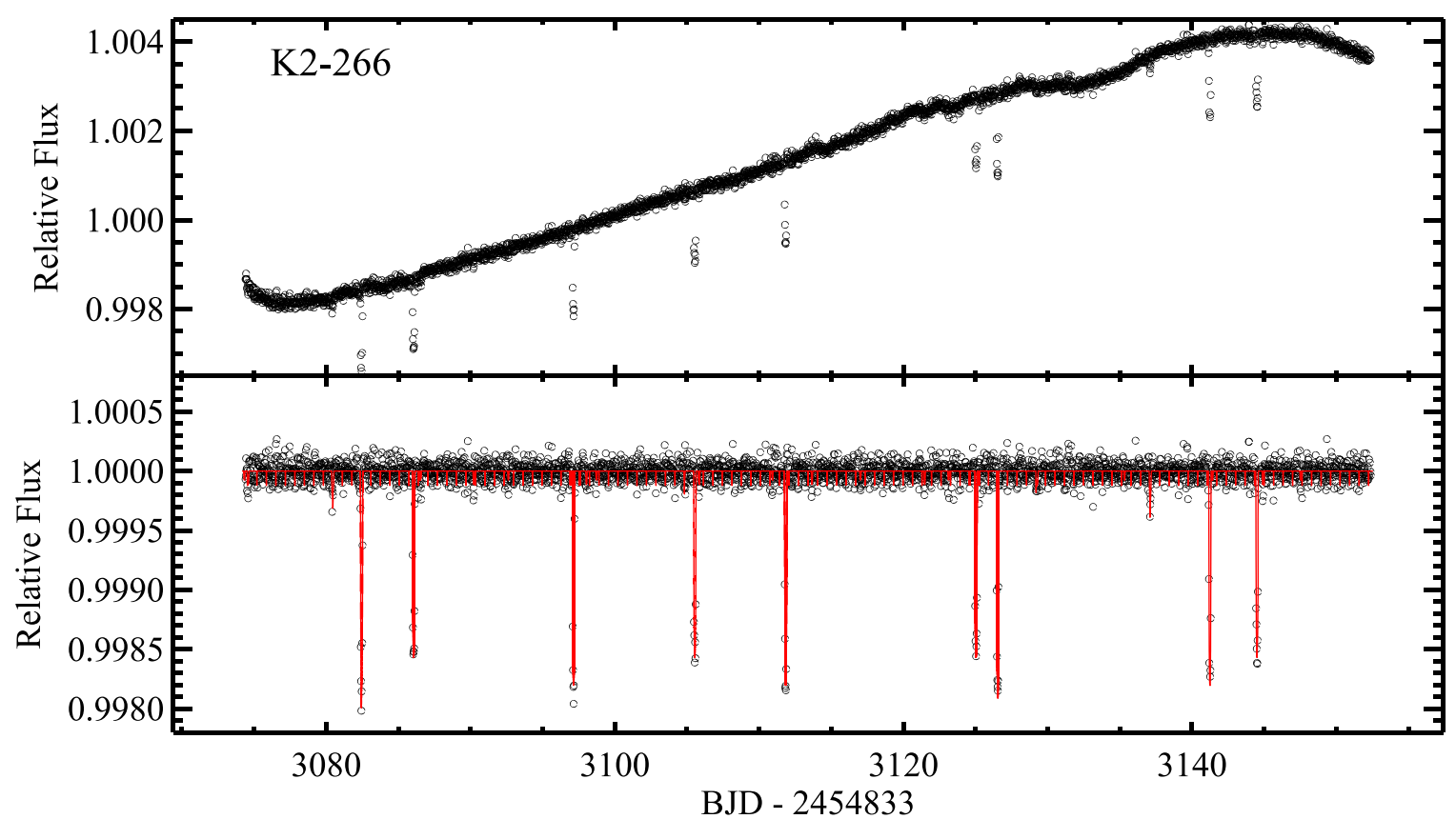

Figure 1. Top panel: the full $K 2$ light curve of K2-266 from Campaign 14, corrected for systematics using the technique described in Vanderburg \& Johnson (2014) and Vanderburg et al. (2016a). The observations are plotted in open black circles, and the best-fit models are plotted in red. Bottom panel: the flattened final $K 2$ light curve used in the EXOFASTv2 fit.

Section 4. Finally, we discuss our results in Section 6 and conclusions in Section 7.

\section{Observations, Archival Data, and Validation}

\subsection{K2 Photometry}

Since the failure of the second reaction wheel, the Kepler spacecraft has been re-purposed to observe a set of fields along the ecliptic. Each $K 2$ campaign lasts $\sim 80$ days (Howell et al. 2014), achieving similar precision to the original Kepler mission (Vanderburg et al. 2016a). K2-266 was observed during K2 Campaign 14 from UT 2017 June 02 until UT 2017 August 19, obtaining 3504 observations on a 30 minute cadence (see Figure 1). Following the strategy described in Vanderburg \& Johnson (2014) and Vanderburg et al. (2016a), the light curves were extracted from the Kepler pipeline calibrated target pixel files from the Mikulski Archive for Space Telescopes, ${ }^{19}$ corrected for the $K 2$ spacecraft-motioninduced systematics, and searched for transiting planet candidates. From our search of K2-266, we identified three super-Earth/sub-Neptune-sized transiting exoplanet candidates with periods of $0.66,14.7$, and 19.5 days, with signal-to-noise $(\mathrm{S} / \mathrm{N})$ values of 13.0, 114.6, and 111.5. In addition, some of us (M.H.K., M.O., H.M.S., and I.T.) performed a visual inspection of the light curve using the LCTOOLS ${ }^{20}$ software (Kipping et al. 2015). From this visual inspection, we identified two additional Earth-sized exoplanet candidates with respective periods of 6.1 and 7.8 days and $S / N$ values of 8.3 and 10.6. An additional visual inspection of the $K 2$ light curve led to the identification of a sixth planet candidate at 56.7 days with an $\mathrm{S} / \mathrm{N}$ value 6.6. The phase-folded light curves for each planet candidate is shown in Figure 2. We note that the two transits of

\footnotetext{
19 MAST; https://archive.stsci.edu/.

${ }^{20}$ https://sites.google.com/a/lctools.net/lctools/home
}

this candidate overlap with other candidates in the system. The $K 2$ light curve was reprocessed where all six planets were simultaneously fit along with the stellar variability and known $K 2$ systematics. The corresponding light curve was flattened by dividing out the best-fit stellar variability using a spline fit with breakpoints every 0.75 days. The final light curve for K2-266, shown in Figure 1, has a 30 minute cadence noise level of $70 \mathrm{ppm}$, and a 6 hour photometric precision of $19 \mathrm{ppm}$.

\subsection{TRES Spectroscopy}

Using the Tillinghast Reflector Echelle Spectrograph (TRES; Fúrész 2008) ${ }^{21}$ on the $1.5 \mathrm{~m}$ Tillinghast Reflector at the Fred L. Whipple Observatory (FLWO) on Mt. Hopkins, AZ we obtained eight observations of K2-266 between UT 2017 November 23 and UT 2018 April 10. TRES has a resolving power of $\lambda / \Delta \lambda=44000$ and an instrumental radial velocity (RV) stability of $10-15 \mathrm{~m} \mathrm{~s}^{-1}$. The spectra were optimally extracted, wavelength calibrated, and cross-correlated to derive relative RVs following the techniques described in Buchhave et al. (2010). We cross-correlate each spectrum, order by order, against the strongest observed spectrum, and fit the peak of the cross-correlation function summed across all orders to derive the relative RVs. Uncertainties are determined from the scatter between orders for each spectrum. We use RV standard stars to track the instrumental zero point over time, and apply these zero point shifts (typically $<15 \mathrm{~m} \mathrm{~s}^{-1}$ ) to the relative RVs and propagate uncertainties in the zero point shifts to the RVs. This is why the strongest spectrum, correlated against itself, does not have an $\mathrm{RV}$ of $0 \mathrm{~m} \mathrm{~s}^{-1}$. The final relative $\mathrm{RVs}$ are given in Table 2. Using the RV standards to set the absolute zero point of the TRES system, we also determine the RV of K2-266 on the IAU standard system to be $10.848 \pm 0.066 \mathrm{~km} \mathrm{~s}^{-1}$, where

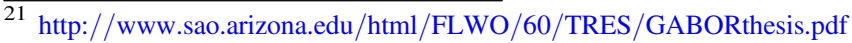



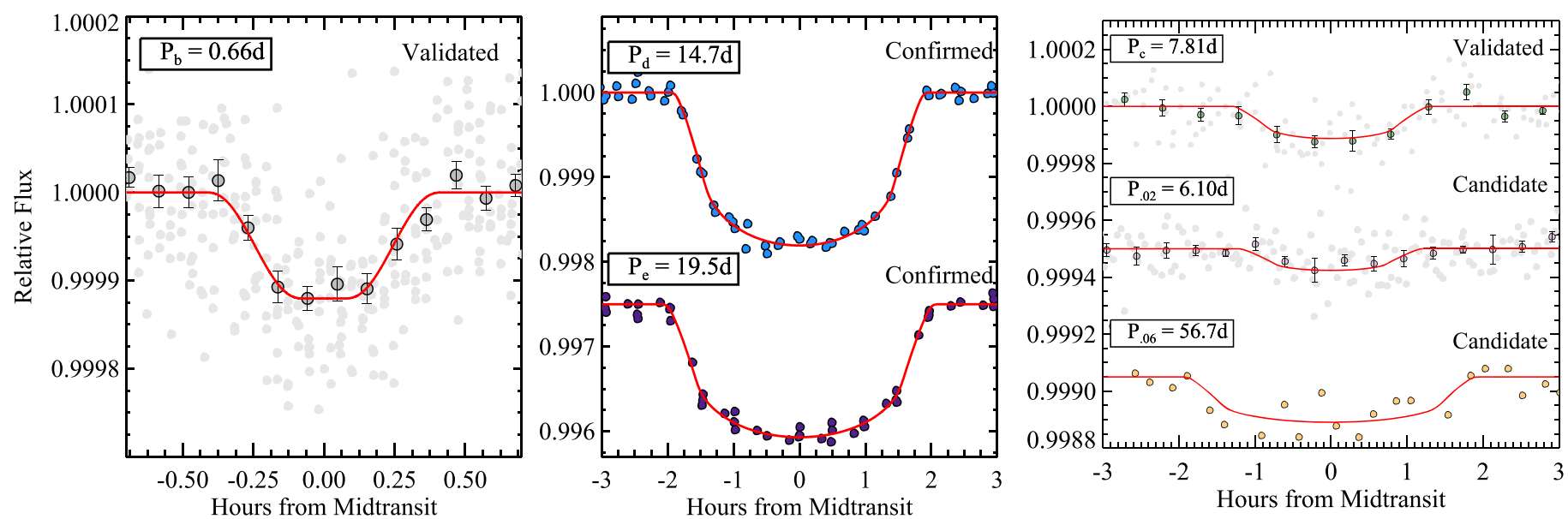

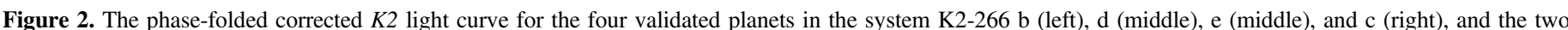

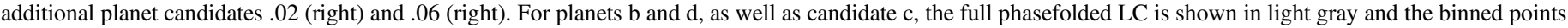
are shown in color with error bars. The red line corresponds to the final EXOFASTv2 transit model.

the uncertainty is dominated by the uncertainty in the shift from relative to absolute $\mathrm{RV}$.

\subsection{Palomar TripleSpec Observations}

We refined the characterization of K2-266 by acquiring nearinfrared spectra using TripleSpec on the 200" Palomar Hale telescope on 2017 December 1. TripleSpec has a fixed slit of $1^{\prime \prime} \times 30^{\prime \prime}$ slit, enabling simultaneous observations across the $\mathrm{J}$, $\mathrm{H}$, and $\mathrm{K}$ bands (1.0-2.4 microns) at a spectral resolution of 2500-2700 (Herter et al. 2008). Following Muirhead et al. (2014), we obtained our observations using a four-position ABCD pattern to reduce the influence of bad pixels on our resulting spectra. As in Dressing et al. (2017), we reduced our data using a version of the publicly available spextool pipeline (Cushing et al. 2004) that was modified for use with TripleSpec data (available upon request from M. Cushing). We removed telluric contamination by observing an A0V star at a similar airmass and processing both our observations for both the A0V star and K2-266 with the xtellcor telluric correction package (Vacca et al. 2003).

After reducing the spectra, we estimated stellar properties by applying empirical relations developed by Newton et al. (2014, 2015) and Mann et al. (2013b, 2013a). Specifically, we estimated the stellar effective temperature and radius by measuring the widths of $\mathrm{Al}$ and $\mathrm{Mg}$ features using the publicly available, IDL-based tellrv and nirew packages (Newton et al. 2014, 2015). We then employed the stellar effective temperature-mass relation developed by Mann et al. (2013a) to infer the stellar mass from the resulting stellar effective temperature estimate. We also estimated the stellar metallicity $([\mathrm{M} / \mathrm{H}]$ and $[\mathrm{Fe} / \mathrm{H}])$ using the relations developed by Mann et al. (2013b). For more details about our TripleSpec analysis methods, see Dressing et al. (2017) and C. D. Dressing et al. (2018, in preparation).

The resulting stellar properties were $T_{\text {eff }}=4192 \pm 77 \mathrm{~K}$, $M_{\star}=0.67_{-0.07}^{+0.08} M_{\odot}$, and $R_{\star}=0.63 \pm 0.03$. These values for the stellar mass are consistent with those estimated from our EXOFASTv2 analysis (see Table 3). However, the radius is $\sim 3 \sigma$ different from the EXOFASTv2 fit using the broadband photometry and Gaia DR2 parallax.

\subsection{Archival "Patient" Imaging}

To check for nearby stars (either physically associated companions or coincidental alignments) that may influence our results, we examined archival observations from National Geographic Society Palomar Observatory Sky Survey (NGS POSS) from 1952. The proper motion of K2-266 is $\mu_{\alpha}=56.9$ mas and $\mu_{\delta}=-68.8$ mas, and has moved $\sim 6^{\prime \prime}$ in the 66 years since the original POSS observations were taken. The present-day position of K2-266 is located right at the edge of the saturated point spread function of K2-266 in the original POSS plates. While the present-day position of K2-266 is not completely resolved in the POSS image, if there was a sufficiently bright background star at the present-day position of K2-266, we would expect to see some elongation of the POSS point spread function at that position. We see no evidence for such an elongation in POSS plates with either a red-sensitive or blue-sensitive emulsion. We estimate that we can rule out background stars at the present-day position of K2-266 down to a magnitude of about 19 in blue, and a magnitude of about 18 in the red. Figure 3 shows our archival imaging overlaid with the $K 2$ photometric aperture used to extract the light curves.

We used modern imaging from the Pan-STARRS data release to search for faint companions at distances greater than a few arcseconds from K2-266 (Flewelling et al. 2016). In the Pan-STARRS images, we identified one star located inside our best photometric aperture about nine magnitudes fainter than $\mathrm{K} 2-266$. In principle, if this star were a fully eclipsing binary (with $100 \%$ deep eclipses ${ }^{22}$ ), it could contribute a transit-like signal to the light curve of K2-266 with a depth of at most about $250 \mathrm{ppm}$. This is shallower than the transits of the two sub-Neptunes, but could in principle contribute the transits of the other four candidates. We therefore extracted the $K 2$ light curve from a smaller aperture (shown in Figure 3 as a navy blue outline overlaid on the Pan-STARRS image of K2-266), which excludes the companion star detected in Pan-STARRS

\footnotetext{
${ }^{22}$ While the greatest eclipse depth possible from two main-sequence eclipsing binaries is about $50 \%$ (caused by an equal-brightness binary), we also consider the worst-case scenario of $100 \%$ deep eclipses caused by, for example, a bright, hot white dwarf being eclipsed by a cool M-dwarf or brown dwarf (e.g., Rappaport et al. 2017).
} 

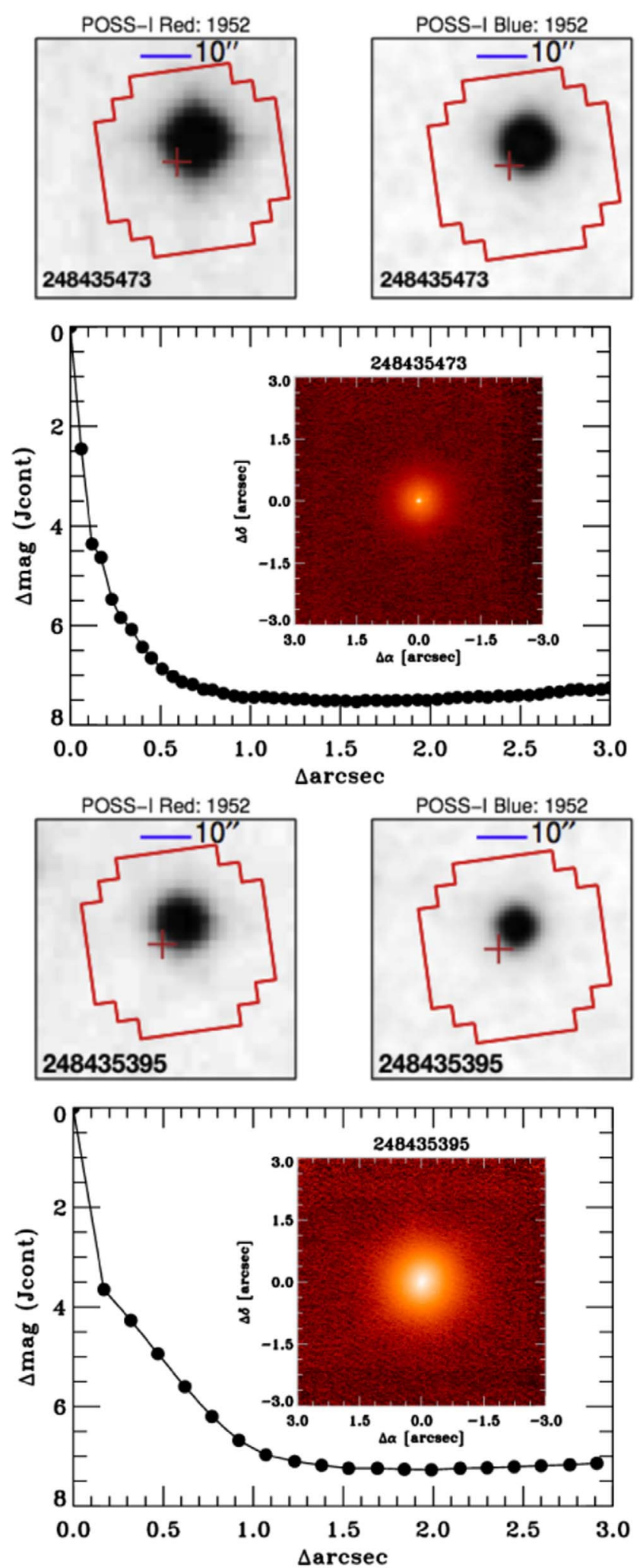
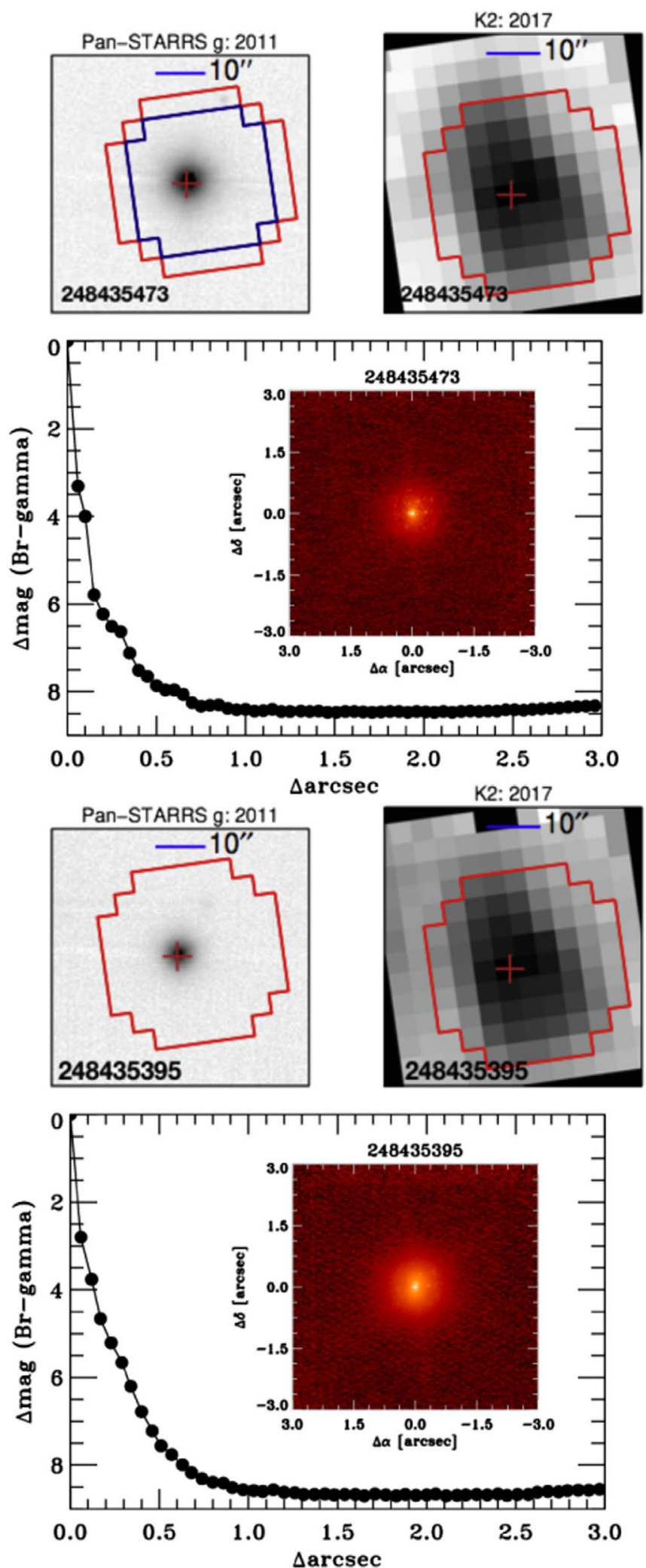

Figure 3. First row: archival imaging from the National Geographic Society Palomar Observatory Sky Survey (NGS POSS) of K2-266 taken with a red (first panel) and blue (second panel) emulsion in 1952. Third panel: archival imaging from the Pan-STARRS survey of K2-266 taken in 2011. Top right: summed image of K2-266 from $K 2$ observations. The aperture selection is described in Vanderburg et al. (2016a). Bottom row: the same four images for EPIC 248435395. The Keck $J$-band (left) and $\mathrm{Br}-\gamma$ contrast curve (right) and image (inset) of K2-266 (second row) and EPIC 248435395 (third row). We note that the J-band image for EPIC 248435395 was observed through poorer atmospheric conditions, leading to reduced image quality. We find no evidence of any additional components in either system. 
imaging. In the noisier light curve extracted from the smaller aperture, we find that the transits of the USP and 7.8 day planets are convincingly detected, but the transits of the two weaker candidates (at 6.1 and 56.7 days) do not convincingly appear (due to the increased noise in the light curve). We therefore cannot rule out a blended background eclipsing binary origin for at least one of those candidates.

\subsection{Keck/NIRC2 AO Imaging}

We obtained high-resolution images of K2-266 using the Near Infrared Camera 2 (NIRC2) on the W. M. Keck Observatory. Two observations of each target were taken on UT 2017 December 28, one in the Br- $\gamma$ filter and the other in the $J$-band (see Figure 3 ). NIRC2 has 9.942 mas pix $^{-1}$ pixel scale and $1024 \times 1024$ pixel array. The lower left quadrant of the array suffers from higher noise levels. To exclude this part of the detector, a three-point dither pattern was used. The final image shown in Figure 3 is created by shifting and co-adding the observations, after flat-fielding and sky subtraction. We see no other star in the $10^{\prime \prime}$ field of view for K2-266. Our sensitivity to nearby companions is determined by injecting a simulated source with an $\mathrm{S} / \mathrm{N}$ of 5 . The final $5 \sigma$ sensitivity curves as a function of spatial separation and the corresponding images for K2-266 in both Br- $\gamma$ and $J$ filters are shown in Figure 3.

\subsection{Statistical Validation}

We attempted to statistically validate each of the six candidates in K2-266, a process in which the probability of planethood is estimated. If the probability is above some threshold value, the candidate is upgraded to a validated planet. Our method of validation followed the approach taken by Mayo et al. (2018). In detail, we made use of vespa (Morton 2015), a Python package based on the work of Morton (2012). The vespa package calculates the false positive probability (FPP) of an exoplanet candidate by first simulating a population of synthetic stellar systems, each of which creates a transit signal due to a planet or eclipsing binary scenario. Then, vespa calculates the FPP by determining which synthetic systems are consistent with the input observations and calculating the fraction of those systems that correspond to an eclipsing binary scenario.

This determination is made based on inputs such as the sky position of the target, the transit signal, various stellar parameters, and contrast curves from any available highresolution imaging. (A contrast curve relates the angular separation between the target star and an undetected companion to the maximum brightness for the putative companion.) In the case of K2-266, we provided as input to vespa the R.A. and decl., the phase-folded light curve of the candidate in question (with transits from other candidates removed), $J, H$, and $K_{S}$ bandpass stellar magnitudes from 2MASS (Cutri et al. 2003; Skrutskie et al. 2006) and the Kepler magnitude, stellar parameters $\left(T_{\text {eff }}, \log g\right.$, and $\left.[\mathrm{Fe} / \mathrm{H}]\right)$ calculated in Section 3, and contrast curves from two AO images.

After we subjected each of our six candidates to validation, we made two additional adjustments to their FPP estimates. First, there are eight spectra and corresponding RV measurements collected with TRES from 2017 November 23 to 2018 April 10. The RV measurements derived from the TRES spectra did not display any large variations indicative of a simple eclipsing binary, so we were able to eliminate that scenario. (Note that this is different from a background eclipsing binary or hierarchical eclipsing binary scenario, which we also consider.) By eliminating the possibility of a simple eclipsing binary, the probability of the planet scenario (and each false positive scenario) was increased such that the total probability remained at unity.

Second, according to Lissauer et al. (2012), the likelihood of one or more false positives decreases significantly when there is more than one candidate in a system. In the case of a system with more than two candidates, they estimate that a multiplicity boost factor of 50 is appropriate. As a result, we decreased the FPP for each candidate by a factor of 50 .

After calculating FPP values for our six candidates, reducing the eclipsing binary scenario to zero probability, and including a multiplicity boost of 50 , we found respective final FPP values of $3.02 e-05,7.34 e-06,9.40 e-06,6.80 e-11,1.16 e-12$, and $4.90 e-06$ for candidates $\mathrm{K} 2-266.01, .02, .03, .04, .05$, and .06 . These values would each be low enough to easily validate all six candidates (e.g., Mayo et al. (2018) used an FPP threshold value of $1 e-4)$. However, given that we cannot rule out the possibility that the faint background star we identified in Section 2.4 is an eclipsing binary, we were only able to conclusively validate candidates $\mathrm{K} 2-266.01, .03, .04$, and .05, naming them K2-266 b, c, d, and e, respectively. We also refrain from validating candidates .02 and .06 because they have the lowest signal-to-noise ratios that do not pass our threshold (8.3 and 6.6, respectively). Validating such low-S/N candidates is challenging because it is difficult to prove that the weakest signals detected in Kepler or $K 2$ data are astrophysical, and not the result of residual instrumental systematics or artifacts (Mullally et al. 2018).

\section{EXOFASTv2 Global Fit for K2-266}

Using the global exoplanet fitting suite, EXOFASTv2 (Eastman 2017), we perform a simultaneous fit of the existing observations to determine the final system parameters for K2266. Based largely on the original EXOFAST (Eastman et al. 2013), EXOFASTv2 provides the unique flexibility to simultaneously fit the spectral energy distribution (SED) and RV observations from multiple instruments, in combination with fitting the time series photometry for every planet in the system. Using EXOFASTv2, we simultaneously fit the flattened $K 2$ light curve (accounting for the 30 minute cadence smearing; see Figures 1 and 2), the SED (see Table 1), and the radial velocity observations from TRES (see Figure 5). To characterize the host star radius within the fit, we include the the broadband photometry and Gaia DR2 parallax (see Table 1) (Gaia Collaboration et al. 2016, 2018). We add 0.082 mas to the DR2 parallax, as determined by Stassun \& Torres (2018) and impose a systematic error floor on the uncertainty of 0.1 mas because all systematics and uncertainties should be below this (Gaia Collaboration et al. 2018). To constrain the mass of the star, we used a Gaussian prior of $0.677 \pm 0.034 M_{\odot}$ from Mann et al. (2015), but with the uncertainties inflated to $5 \%$. In a separate global fit (not reported), we used the MIST stellar isochrones (Paxton et al. 2011, 2013, 2015; Choi et al. 2016; Dotter 2016) instead of the Gaussian prior from Mann et al. (2015) as the primary constraint of the stellar mass and arrived at $0.748_{-0.045}^{+0.047} M_{\odot}$, a $1.3 \sigma$ difference. We favor the Mann et al. (2015) relations due to their empirical approach and the known problems with all model isochrones at low stellar masses. 

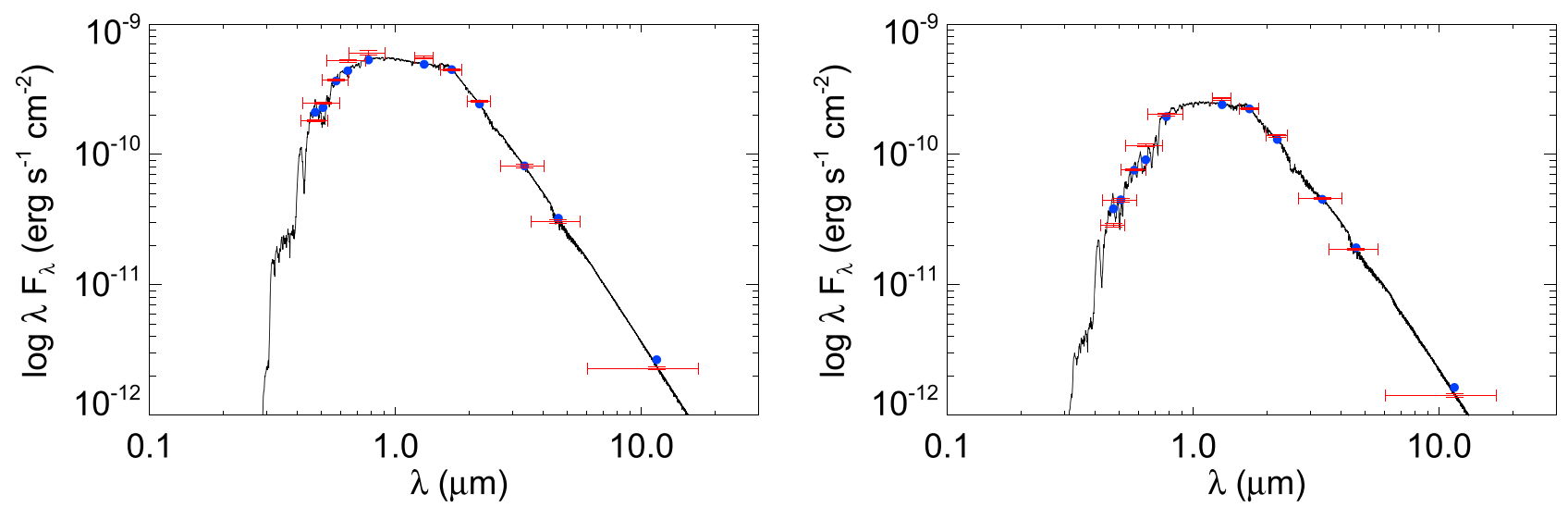

Figure 4. The SED fit for (left) K2-266 and (right) EPIC 248435395 from EXOFASTv2. The blue points are the predicted integrated fluxes and the red points are the observed values at the corresponding passbands. The width of the bandpasses are the horizontal red error bars and the vertical errors represent the $1 \sigma$ uncertainties. The final model fit is shown by the solid line.

Table 1

EPIC 248435473 and EPIC 248435395 Magnitudes and Kinematics

\begin{tabular}{|c|c|c|c|c|}
\hline \multicolumn{5}{|c|}{$\begin{array}{c}\text { 2MASS } 10314174+0056048 \\
\text { EPIC } 248435395\end{array}$} \\
\hline Parameter & Description & Value & Value & Source \\
\hline$\alpha_{J 2000}$ & Right Ascension (R.A.) & 10:31:44.506 & $10: 31: 41.749$ & 1 \\
\hline$\delta_{J 2000}$ & Declination (Decl.) & $+00: 56: 15.27$ & $+00: 56: 04.94$ & 1 \\
\hline$B^{\mathrm{a}}$ & APASS Johnson $B$ mag & $13.001 \pm 0.02$ & $15.011 \pm 0.04$ & 2 \\
\hline$g^{\prime}$ & APASS Sloan $g^{\prime}$ mag & $12.407 \pm 0.02$ & $14.270 \pm 0.03$ & 2 \\
\hline$r^{\prime}$ & APASS Sloan $r^{\prime}$ mag & $11.311 \pm 0.02$ & $12.931 \pm 0.03$ & 2 \\
\hline$i^{\prime}$ & APASS Sloan $i^{\prime}$ mag & $10.927 \pm 0.04$ & $12.111 \pm 0.03$ & 2 \\
\hline$J$ & 2MASS $J$ mag & $9.611 \pm 0.05$ & $10.405 \pm 0.03$ & 3,4 \\
\hline$H$ & 2MASS $H$ mag & $9.041 \pm 0.03$ & $9.784 \pm 0.02$ & 3,4 \\
\hline$\mu_{\alpha}$ & $\begin{array}{l}\text { Gaia DR2 proper motion } \\
\text { in R.A. }\left(\mathrm{mas}^{-1}\right)\end{array}$ & $56.871 \pm 0.151$ & $53.231 \pm 0.161$ & 7,8 \\
\hline$\mu_{\delta}$ & $\begin{array}{l}\text { Gaia DR2 proper motion } \\
\text { in Decl. }\left(\mathrm{mas} \mathrm{yr}^{-1}\right)\end{array}$ & $-68.828 \pm 0.242$ & $-72.735 \pm 0.263$ & 7,8 \\
\hline$\pi$ & Gaia Parallax (mas) & $12.87 \pm 0.06$ & $12.85 \pm 0.06$ & 7,8 \\
\hline RV & $\begin{array}{l}\text { Systemic radial } \\
\text { velocity }\left(\mathrm{km} \mathrm{s}^{-1}\right)\end{array}$ & $10.848 \pm 0.066$ & $12.84 \pm 0.31$ & Sections $2.2,5$ \\
\hline
\end{tabular}

Note. References: (1) Cutri et al. (2003), (2) Henden et al. (2016), (3) Cutri et al. (2003), (4) Skrutskie et al. (2006), (5) Cutri et al. (2014), (6) Zacharias et al. (2017), (7) Gaia Collaboration et al. (2016), (8) Gaia Collaboration et al. (2018).

${ }^{a}$ The uncertainties of the photometry have a systematic error floor applied. Even still, the global fit requires a significant scaling of the uncertainties quoted here to be consistent with our model, suggesting they are still significantly underestimated for one or more of the broadband magnitudes.

Additionally, we enforce an upper limit in the $V$-band extinction $\left(A_{V}\right)$ from the Schlegel et al. (1998) dust maps of 0.0548 at the position of K2-266. The final SED fit is shown in Figure 4, the phase-folded RVs from TRES to planet b's period is shown in Figure 5, and the best-fit transit models are shown in Figures 1 and 2. Given the near resonance orbit of K2-266 d and e, which would have the largest transit timing variations (TTVs), we fit for the TTVs of these two planets while fitting a linear ephemeris for planets/candidates b, .02, c, and .06. The final determined stellar and planetary parameters from our global fit are shown in Tables 3-5.

The grazing geometry of planet $b$ means the upper limit of the planet radius is unconstrained by the light curve. However, during the global fit, we simultaneously model the radial velocities, which provide a robust upper limit on its mass. This upper limit is translated to a radius upper limit during the global 


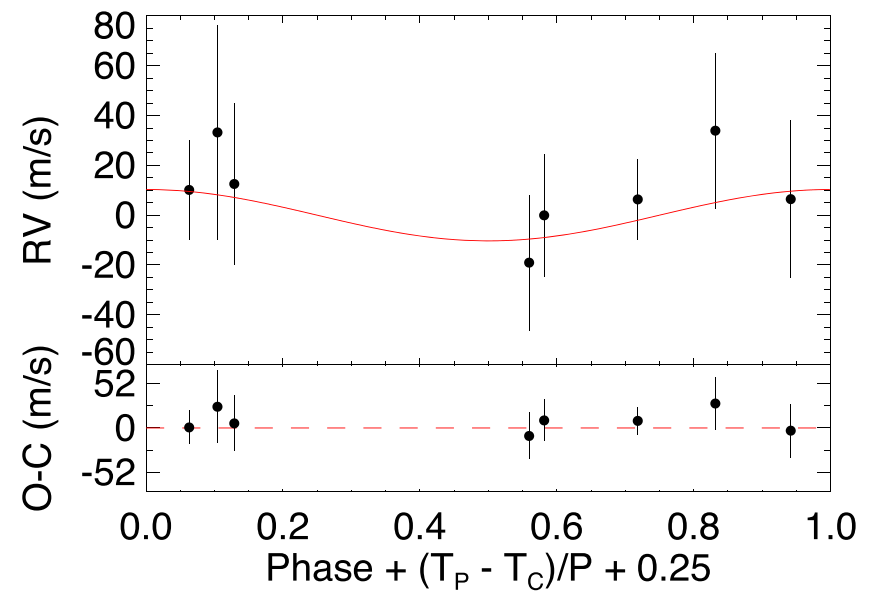

Figure 5. The TRES radial velocity measurements phase-folded to the best-fit ephemeris of K2-266 b. The primary transit occurs at a phase of 0.25 , where $T_{P}$ is the time of periastron, $T_{C}$ is the time of transit, and $P$ period of planet $\mathrm{b}$.

fit using EXOFASTv2's integrated Chen \& Kipping (2017) exoplanet mass-radius relation, which excludes Jupiter-radius solutions (and even higher inclinations). Because the radial velocities are not precise enough for a measurement, the prior, which is uniform in $\log (K)$, can have a significant impact on the posteriors for the RV semi-amplitude, mass, radius, and inclination of planet $\mathrm{b}$ and tends to favor smaller planets and smaller inclinations (in line with our prior expectation that such planets are intrinsically more numerous).

Because the Chen \& Kipping (2017) relations only use a sample of planets with robustly detected masses and radii, and we can typically measure robust radii for smaller planets than we can measure the corresponding masses, there is likely a selection effect in the Chen \& Kipping (2017) relations that bias it toward larger masses at low signal to noise. As a consequence, for a given mass, we expect to overestimate the radius (see Figure 6 for the probability distribution for the radius of planet b). Our radius upper limit would likely be somewhat smaller if we used a relation that accounted for non-detections within our fit.

The Weiss \& Marcy (2014) exoplanet mass-radius relations are an often-used alternative that attempts to account for the bias from non-detections. However, they only apply to rocky planets $\left(R_{p}<4 R_{\oplus}\right)$, and so could not be used to exclude large planets, whereas the Chen \& Kipping (2017) relations are defined and continuous from rocky planets to stars.

\section{Dynamics of K2-266}

Given its multiplicity and mutually transiting nature, the sixplanet system orbiting K2-266 can be classified as one of the Systems of Tightly Packed Inner Planets (STIPs) common in the Kepler data (Lissauer et al. 2011; Van Laerhoven \& Greenberg 2012; Swift et al. 2013). However, this system is unique due to the innermost planetary orbit, displaying a remarkable $75^{\circ}$ inclination and a grazing transit. Members of the Kepler multi-planet systems have smaller mutual inclinations, typically within a few degrees of each other (Fang \& Margot 2012; Figueira et al. 2012; Fabrycky et al. 2014). Moreover, these systems do not tend to excite high mutual inclinations without some external factor (Becker \& Adams 2016, 2017; Hansen 2017; Jontof-Hutter et al. 2017; Mustill et al. 2017; Denham et al. 2018). In this section, we discuss the information gained through combining the observed
Table 2

Relative Radial Velocities for K2-266 and EPIC 248435395

\begin{tabular}{lccl}
\hline \hline $\mathrm{BJD}_{\mathrm{TDB}}$ & $\mathrm{RV}\left(\mathrm{m} \mathrm{s}^{-1}\right)$ & $\sigma_{R V}\left(\mathrm{~m} \mathrm{~s}^{-1}\right)$ & Target \\
\hline 2458081.028322 & -30.1 & 17.7 & $\mathrm{~K} 2-266$ \\
2458090.980928 & -2.5 & 32.0 & $\mathrm{~K} 2-266$ \\
2458106.965261 & -3.2 & 43.6 & $\mathrm{~K} 2-266$ \\
2458107.923621 & -55.5 & 28.2 & $\mathrm{~K} 2-266$ \\
2458211.644609 & -26.3 & 21.2 & $\mathrm{~K} 2-266$ \\
2458212.644636 & -36.5 & 25.6 & $\mathrm{~K} 2-266$ \\
2458213.663584 & -23.9 & 33.3 & $\mathrm{~K} 2-266$ \\
2458218.808648 & -30.0 & 32.5 & $\mathrm{~K} 2-266$ \\
\hline 2458156.911319 & 425.9 & 46.9 & EPIC 248435395 \\
2458211.675712 & -21.6 & 47.9 & EPIC 248435395 \\
\hline
\end{tabular}

Table 3

Median Values and 68\% Confidence Intervals for the Stellar Parameters of the K2-266 and EPIC 248435395 from EXOFASTv2

\begin{tabular}{llll}
\hline \hline Stellar & Parameters & K2-266 & EPIC 248435395 \\
\hline$M_{*}$ & Mass $\left(M_{\odot}\right)$ & $0.686 \pm 0.033$ & $0.581 \pm 0.029$ \\
$R_{*}$ & Radius $\left(R_{\odot}\right)$ & $0.703_{-0.022}^{+0.024}$ & $0.649_{-0.035}^{+0.040}$ \\
$L_{*}$ & Luminosity $\left(L_{\odot}\right)$ & $0.1502 \pm 0.0057$ & $0.0617_{-0.0028}^{+0.0027}$ \\
$\rho_{*}$ & Density (cgs) & $2.79_{-0.30}^{+0.29}$ & $2.99_{-0.51}^{+0.56}$ \\
$\log g$ & Surface gravity (cgs) & $4.581_{-0.037}^{+0.032}$ & $4.577_{-0.056}^{+0.052}$ \\
$T_{\text {eff }}$ & Effective temperature $(\mathrm{K})$ & $4285_{-57}^{+49}$ & $3570 \pm 110$ \\
{$[\mathrm{Fe} / \mathrm{H}]$} & Metallicity & $-0.12_{-0.42}^{+0.40}$ & $-0.19_{-0.81}^{+0.43}$ \\
$A_{v}$ & V-band extinction & $0.029_{-0.019}^{+0.018}$ & $0.028_{-0.019}^{+0.018}$ \\
$\sigma_{\mathrm{SED}}$ & SED photometry error & $5.0_{-1.1}^{+1.6}$ & $5.5_{-1.2}^{+1.8}$ \\
$\pi^{\mathrm{a}}$ & $\quad$ scaling & $12.960 \pm 0.100$ & $12.928 \pm 0.098$ \\
$d$ & Parallax (mas) & $77.16_{-0.59}^{+0.60}$ & $77.35_{-0.58}^{+0.59}$ \\
\hline
\end{tabular}

Note.

${ }^{\mathrm{a}}$ The MIST Isochrones were not used in the EXOFASTv2 fit for K2-266.

light curve with dynamical analysis, and attempt to constrain the current dynamical state of the system.

\subsection{Transit Timing Variations}

The transit timing measurements for planets $d$ and e listed in Table 3 can be used to derive dynamical constraints on their masses and orbits. In this section, we invert the planet pairs' TTVs to infer their masses, and in combination with the planet radii derived from out light-curve fitting, their densities. We model the planets' TTVs using the TTVFast code developed by Deck et al. (2014) and use the emcee package's (ForemanMackey et al. 2013) ensemble sampler, based on the algorithm of Goodman \& Weare (2010), to sample the posterior distribution of the planetary masses and orbital elements. We model only the dynamical interactions of planets $d$ and e, and ignore any perturbations from the other (potential) members of the system. ${ }^{23} \mathrm{We}$ assume planets $\mathrm{d}$ and e orbit in the same plane because small mutual inclinations have negligible influence on TTVs. We approximate the mid-transit

\footnotetext{
23 The variations induced by the additional planets in the system are expected to be negligible. For example, assuming a $1 M_{\oplus}$ planet $\mathrm{c}$ and circular orbits, it induces variations of less than 10 seconds in planet d's transit times. Allowing for modest eccentricities does not significantly enhance the induced TTVs. The influence of the other additional planets is expected to be even weaker, given that they are more widely separated and do not fall near any significant resonances with $\mathrm{d}$ or e.
} 
Table 4

Median Values and $68 \%$ Confidence Intervals for Planetary Parameters of K2-266 from EXOFASTv2

\begin{tabular}{|c|c|c|c|c|c|c|c|}
\hline \multicolumn{2}{|c|}{ Planetary Parameters: } & \multirow{2}{*}{$\begin{array}{l}\mathrm{b} \\
0.658524 \pm 0.000017\end{array}$} & \multirow{2}{*}{$\begin{array}{l}\mathrm{K} 2-266.02 \\
6.1002_{-0.0017}^{+0.0015}\end{array}$} & \multirow{2}{*}{$\begin{array}{l}\mathrm{c} \\
7.8140_{-00016}^{+0.0019}\end{array}$} & \multirow{2}{*}{$\begin{array}{l}\mathrm{d} \\
14.69700_{-0.00035}^{+0.00034}\end{array}$} & \multirow{2}{*}{$\begin{array}{l}\mathrm{e} \\
19.4820 \pm 0.0012\end{array}$} & \multirow{2}{*}{$\begin{array}{l}\mathrm{K} 2-266.06 \\
56.682_{-0.018}^{+0.019}\end{array}$} \\
\hline$P$ & Period (days) & & & & & & \\
\hline$R_{P}$ & Radius $\left(R_{\oplus}\right)$ & $3.3_{-1.3}^{+1.8}$ & $0.646_{-0.091}^{+0.099}$ & $0.705_{-0.085}^{+0.096}$ & $2.93_{-0.12}^{+0.14}$ & $2.73_{-0.11}^{+0.14}$ & $0.90_{-0.12}^{+0.14}$ \\
\hline$T_{C}$ & Time of conjunction $\left(\mathrm{BJD}_{\mathrm{TDB}}\right)$ & $2457945.7235_{-0.0030}^{+0.0032}$ & $2457913.413_{-0.011}^{+0.013}$ & $2457907.5812_{-0.012}^{+0.0099}$ & $2457944.8393 \pm 0.0012$ & $2457938.5410 \pm 0.0013$ & $2457913.436_{-0.013}^{+0.014}$ \\
\hline$T_{0}$ & Optimal conjunction time $\left(\mathrm{BJD}_{\mathrm{TDB}}\right)$ & $2457949.6747_{-0.0030}^{+0.0032}$ & $2457943.9143_{-0.0064}^{+0.0066}$ & $2457946.6510 \pm 0.0064$ & $2457944.8393 \pm 0.0012$ & $2457938.5410 \pm 0.0013$ & $2457913.436_{-0.013}^{+0.014}$ \\
\hline$a$ & Semimajor axis (au) & $0.01306_{-0.00021}^{+0.00020}$ & $0.05761_{-0.00093}^{+0.00090}$ & $0.0679 \pm 0.0011$ & $0.1035_{-0.0017}^{+0.0016}$ & $0.1249_{-0.0020}^{+0.0019}$ & $0.2546_{-0.0041}^{+0.0040}$ \\
\hline$i$ & Inclination (degrees) & $75.32_{-0.70}^{+0.62}$ & $87.84_{-0.46}^{+0.84}$ & $88.28_{-0.41}^{+0.81}$ & $89.46_{-0.25}^{+0.32}$ & $89.45_{-0.18}^{+0.25}$ & $89.40_{-0.14}^{+0.26}$ \\
\hline$e$ & Eccentricity & $\ldots$ & $0.051_{-0.036}^{+0.051}$ & $0.042_{-0.030}^{+0.043}$ & $0.047_{-0.032}^{+0.043}$ & $0.043_{-0.030}^{+0.036}$ & $0.31_{-0.17}^{+0.11}$ \\
\hline$\omega_{*}$ & Argument of periastron (degrees) & $\cdots$ & $88_{-62}^{+60}$ & $87 \pm 61$ & $87 \pm 62$ & $89_{-58}^{+57}$ & $83_{-59}^{+57}$ \\
\hline$T_{\mathrm{eq}}$ & Equilibrium temperature $(\mathrm{K})$ & $1515 \pm 18$ & $721.7_{-8.4}^{+8.7}$ & $664.5_{-7.7}^{+8.0}$ & $538.3_{-6.3}^{+6.5}$ & $490.1_{-5.7}^{+5.9}$ & $343.3_{-4.0}^{+4.1}$ \\
\hline$M_{P}$ & Mass $\left(M_{\oplus}\right)$ & $11.3_{-6.5}^{+11}$ & $0.209_{-0.089}^{+0.15}$ & $0.29_{-0.11}^{+0.17}$ & $9.4_{-2.0}^{+2.9}$ & $8.3_{-1.8}^{+2.7}$ & $0.70_{-0.30}^{+0.87}$ \\
\hline$K$ & RV semi-amplitude $\left(\mathrm{m} \mathrm{s}^{-1}\right)$ & $10.3_{-5.9}^{+10.9}$ & $0.094_{-0.040}^{+0.067}$ & $0.119_{-0.046}^{+0.073}$ & $3.17_{-0.69}^{+0.99}$ & $2.53_{-0.56}^{+0.84}$ & $0.158_{-0.068}^{+0.20}$ \\
\hline $\log K$ & Log of RV semi-amplitude & $1.01_{-0.37}^{+0.31}$ & $-1.03_{-0.24}^{+0.23}$ & $-0.92 \pm 0.21$ & $0.50_{-0.11}^{+0.12}$ & $0.40_{-0.11}^{+0.12}$ & $-0.80_{-0.24}^{+0.35}$ \\
\hline$R_{P} / R_{*}$ & Radius of planet in stellar radii & $0.043_{-0.017}^{+0.023}$ & $0.0084_{-0.0011}^{+0.0012}$ & $0.0092_{-0.0011}^{+0.0012}$ & $0.03827_{-0.00057}^{+0.00071}$ & $0.03564_{-0.00061}^{+0.00067}$ & $0.0117_{-0.0015}^{+0.0018}$ \\
\hline$a / R_{*}$ & Semimajor axis in stellar radii & $4.00_{-0.15}^{+0.14}$ & $17.63_{-0.66}^{+0.60}$ & $20.80_{-0.78}^{+0.71}$ & $31.7_{-1.2}^{+1.1}$ & $38.2_{-1.4}^{+1.3}$ & $77.9_{-2.9}^{+2.7}$ \\
\hline$\delta$ & Transit depth (fraction) & $0.0018_{-0.0012}^{+0.0025}$ & $0.000071_{-0.000018}^{+0.000022}$ & $0.000085_{-0.000019}^{+0.000023}$ & $0.001465_{-0.000043}^{+0.000055}$ & $0.001270_{-0.000043}^{+0.000048}$ & $0.000136_{-0.000033}^{+0.000046}$ \\
\hline Depth & Flux decrement at mid-transit & $0.000596_{-0.000091}^{+0.000068}$ & $0.000071_{-0.000018}^{+0.000022}$ & $0.000085_{-0.000019}^{+0.000023}$ & $0.001465_{-0.000043}^{+0.000055}$ & $0.001270_{-0.000043}^{+0.000048}$ & $0.000136_{-0.000033}^{+0.000046}$ \\
\hline$\tau$ & Ingress/egress transit duration (days) & $0.00686_{-0.00045}^{+0.00051}$ & $0.00116_{-0.00032}^{+0.00042}$ & $0.00134_{-0.00033}^{+0.00045}$ & $0.00569_{-0.00045}^{+0.00067}$ & $0.00601_{-0.00063}^{+0.00073}$ & $0.00281_{-0.00096}^{+0.0019}$ \\
\hline$T_{14}$ & Total transit duration (days) & $0.01389_{-0.00085}^{+0.00012}$ & $0.083_{-0.012}^{+0.014}$ & $0.094_{-0.014}^{+0.016}$ & $0.1420_{-0.0015}^{+0.0014}$ & $0.1527_{-0.0015}^{+0.0014}$ & $0.143_{-0.023}^{+0.024}$ \\
\hline$T_{\mathrm{FWHM}}$ & FWHM transit duration (days) & $0.00695_{-0.00042}^{+0.00062}$ & $0.082_{-0.013}^{+0.015}$ & $0.092_{-0.014}^{+0.016}$ & $0.1362 \pm 0.0015$ & $0.1466_{-0.0016}^{+0.0015}$ & $0.140 \pm 0.024$ \\
\hline$b$ & Transit impact parameter & $1.011_{-0.024}^{+0.027}$ & $0.64_{-0.25}^{+0.13}$ & $0.60_{-0.28}^{+0.14}$ & $0.29_{-0.17}^{+0.12}$ & $0.36_{-0.16}^{+0.10}$ & $0.64_{-0.33}^{+0.17}$ \\
\hline$b_{S}$ & Eclipse impact parameter & $\ldots$ & $0.68_{-0.26}^{+0.13}$ & $0.64_{-0.30}^{+0.14}$ & $0.31_{-0.18}^{+0.11}$ & $0.379_{-0.16}^{+0.100}$ & $0.86_{-0.33}^{+0.30}$ \\
\hline$\tau_{S}$ & Ingress/egress eclipse duration (days) & $\ldots$ & $0.00131_{-0.00038}^{+0.00055}$ & $0.00148_{-0.00039}^{+0.00057}$ & $0.00613_{-0.00048}^{+0.00064}$ & $0.00641_{-0.00052}^{+0.00069}$ & $0.0037_{-0.0037}^{+0.0035}$ \\
\hline$T_{S, 14}$ & Total eclipse duration (days) & $\cdots$ & $0.084_{-0.016}^{+0.019}$ & $0.096_{-0.017}^{+0.020}$ & $0.1485_{-0.0051}^{+0.0100}$ & $0.1593_{-0.0052}^{+0.010}$ & $0.13_{-0.13}^{+0.10}$ \\
\hline$T_{S, \text { FWHM }}$ & FWHM eclipse duration (days) & $\ldots$ & $0.083_{-0.017}^{+0.020}$ & $0.094_{-0.018}^{+0.021}$ & $0.1424_{-0.0052}^{+0.0099}$ & $0.1528_{-0.0053}^{+0.011}$ & $0.13_{-0.13}^{+0.11}$ \\
\hline$\delta_{S, 3.6 \mu \mathrm{m}}$ & Blackbody eclipse depth at $3.6 \mu \mathrm{m}$ (ppm) & $210_{-140}^{+290}$ & $0.41_{-0.11}^{+0.14}$ & $0.299_{-0.069}^{+0.088}$ & $1.24_{-0.12}^{+0.15}$ & $0.511_{-0.057}^{+0.070}$ & $0.00160_{-0.00044}^{+0.00062}$ \\
\hline$\delta_{S, 4.5 \mu \mathrm{m}}$ & Blackbody eclipse depth at $4.5 \mu \mathrm{m}(\mathrm{ppm})$ & $280_{-180}^{+380}$ & $0.94_{-0.24}^{+0.31}$ & $0.76_{-0.17}^{+0.22}$ & $4.25_{-0.35}^{+0.44}$ & $2.05_{-0.19}^{+0.24}$ & $0.0135_{-0.0036}^{+0.0050}$ \\
\hline$\rho_{P}$ & Density (cgs) & $1.77_{-0.88}^{+1.9}$ & $4.27_{-0.66}^{+0.79}$ & $4.51_{-0.67}^{+0.81}$ & $2.03_{-0.43}^{+0.64}$ & $2.21_{-0.47}^{+0.70}$ & $5.27_{-0.87}^{+1.5}$ \\
\hline $\log g_{P}$ & Surface gravity & $3.00_{-0.13}^{+0.14}$ & $2.69 \pm 0.12$ & $2.75 \pm 0.11$ & $3.026_{-0.100}^{+0.12}$ & $3.03_{-0.10}^{+0.12}$ & $2.93_{-0.12}^{+0.18}$ \\
\hline$\Theta$ & Safronov Number & $0.0046_{-0.0015}^{+0.0018}$ & $0.00191_{-0.00065}^{+0.00094}$ & $0.00285_{-0.00086}^{+0.0012}$ & $0.0340_{-0.0072}^{+0.010}$ & $0.0387_{-0.0082}^{+0.012}$ & $0.0203_{-0.0070}^{+0.017}$ \\
\hline$\langle F\rangle$ & Incident flux $\left(10^{9} \mathrm{erg} \mathrm{s}^{-1} \mathrm{~cm}^{-2}\right)$ & $1.197_{-0.055}^{+0.059}$ & $0.0612_{-0.0028}^{+0.0031}$ & $0.0441_{-0.0020}^{+0.0022}$ & $0.01898_{-0.00086}^{+0.00093}$ & $0.01304_{-0.00060}^{+0.00064}$ & $0.00286_{-0.00024}^{+0.00025}$ \\
\hline$T_{P}$ & Time of periastron $\left(\mathrm{BJD}_{\mathrm{TDB}}\right)$ & $2457945.7235_{-0.0030}^{+0.0032}$ & $2457913.43_{-0.95}^{+0.93}$ & $2457907.6 \pm 1.2$ & $2457944.8 \pm 2.3$ & $2457938.6 \pm 2.9$ & $2457913.3_{-5.2}^{+5.0}$ \\
\hline$T_{S}$ & Time of eclipse (BJD $\left.\mathrm{BDB}_{\mathrm{TDB}}\right)$ & $2457946.0528_{-0.0030}^{+0.0032}$ & $2457916.46 \pm 0.17$ & $2457903.67_{-0.18}^{+0.19}$ & $2457937.49_{-0.40}^{+0.41}$ & $2457948.28 \pm 0.44$ & $2457885.2_{-8.7}^{+8.6}$ \\
\hline
\end{tabular}


Table 4

(Continued)

\begin{tabular}{|c|c|c|c|c|c|c|c|}
\hline \multicolumn{2}{|c|}{ Planetary Parameters: } & \multirow{2}{*}{$\begin{array}{l}\mathrm{b} \\
2457945.5589_{-0.0030}^{+0.0032}\end{array}$} & \multirow{2}{*}{$\frac{\mathrm{K} 2-266.02}{2457911.942_{-0.076}^{+0.13}}$} & \multirow{2}{*}{$\begin{array}{l}\mathrm{c} \\
2457905.682_{-0.080}^{+0.14}\end{array}$} & \multirow{2}{*}{$\begin{array}{l}\mathrm{d} \\
2457941.28_{-0.17}^{+0.28}\end{array}$} & \multirow{2}{*}{$\begin{array}{l}\mathrm{e} \\
2457933.82_{-0.19}^{+0.32}\end{array}$} & \multirow{2}{*}{$\begin{array}{l}\mathrm{K} 2-266.06 \\
2457903.3_{-4.2}^{+3.4}\end{array}$} \\
\hline$\overline{T_{A}}$ & Time of ascending node $\left(\mathrm{BJD}_{\mathrm{TDB}}\right)$ & & & & & & \\
\hline$T_{D}$ & Time of descending node $\left(\mathrm{BJD}_{\mathrm{TDB}}\right)$ & $2457945.8881_{-0.0030}^{+0.0032}$ & $2457914.884_{-0.13}^{+0.077}$ & $2457909.478_{-0.14}^{+0.078}$ & $2457948.40_{-0.28}^{+0.17}$ & $2457943.26_{-0.33}^{+0.19}$ & $2457923.6_{-3.5}^{+4.3}$ \\
\hline$e \cos \omega_{*}$ & & $\cdots$ & $-0.000_{-0.045}^{+0.044}$ & $0.000 \pm 0.037$ & $-0.000 \pm 0.043$ & $-0.000_{-0.035}^{+0.036}$ & $0.00 \pm 0.24$ \\
\hline$e \sin \omega_{*}$ & & $\ldots$ & $0.026_{-0.022}^{+0.046}$ & $0.022_{-0.018}^{+0.038}$ & $0.025_{-0.020}^{+0.035}$ & $0.025_{-0.020}^{+0.034}$ & $0.19_{-0.15}^{+0.16}$ \\
\hline$V / V_{C}$ & & $\cdots$ & $0.973_{-0.044}^{+0.022}$ & $0.978_{-0.037}^{+0.018}$ & $0.975_{-0.034}^{+0.020}$ & $0.975_{-0.033}^{+0.020}$ & $0.80_{-0.12}^{+0.15}$ \\
\hline$M_{P} \sin i$ & Minimum mass $\left(M_{\oplus}\right)$ & $10.9_{-6.3}^{+11}$ & $0.208_{-0.089}^{+0.15}$ & $0.29_{-0.11}^{+0.17}$ & $9.4_{-2.0}^{+2.9}$ & $8.3_{-1.8}^{+2.7}$ & $0.70_{-0.30}^{+0.87}$ \\
\hline$M_{P} / M_{*}$ & Mass ratio & $0.000049_{-0.000028}^{+0.000051}$ & $0.00000091_{-0.00000039}^{+0.00000065}$ & $0.00000126_{-0.00000049}^{+0.00000077}$ & $0.0000413_{-0.0000091}^{+0.000013}$ & $0.0000362_{-0.0000081}^{+0.000012}$ & $0.0000031_{-0.0000013}^{+0.0000039}$ \\
\hline$d / R_{*}$ & Separation at mid-transit & $4.00_{-0.15}^{+0.14}$ & $16.98_{-0.92}^{+0.81}$ & $20.17_{-1.0}^{+0.87}$ & $30.7_{-1.7}^{+1.4}$ & $37.0_{-1.7}^{+1.6}$ & $59_{-11}^{+13}$ \\
\hline$P_{T}$ & A priori non-grazing transit prob & $0.2389_{-0.0092}^{+0.010}$ & $0.0584_{-0.0027}^{+0.0033}$ & $0.0491_{-0.0020}^{+0.0026}$ & $0.0313_{-0.0014}^{+0.0018}$ & $0.0261_{-0.0011}^{+0.0012}$ & $0.0166_{-0.0030}^{+0.0039}$ \\
\hline$P_{T, G}$ & A priori transit prob & $0.2616_{-0.0098}^{+0.011}$ & $0.0594_{-0.0027}^{+0.0034}$ & $0.0500_{-0.0021}^{+0.0026}$ & $0.0338_{-0.0015}^{+0.0020}$ & $0.0280_{-0.0012}^{+0.0013}$ & $0.0170_{-0.0030}^{+0.0039}$ \\
\hline$P_{S}$ & A priori non-grazing eclipse prob & .. & $0.0546 \pm 0.0025$ & $0.0465 \pm 0.0020$ & $0.02941_{-0.00093}^{+0.0013}$ & $0.0245_{-0.0010}^{+0.0012}$ & $0.0113_{-0.0016}^{+0.0017}$ \\
\hline$P_{S, G}$ & A priori eclipse prob & $\ldots$ & $0.0555 \pm 0.0026$ & $0.0473_{-0.0020}^{+0.0021}$ & $0.0317_{-0.0010}^{+0.0015}$ & $0.0263_{-0.0012}^{+0.0013}$ & $0.0116_{-0.0017}^{+0.0018}$ \\
\hline
\end{tabular}


Table 5

Median Values and 68\% Confidence Intervals for the Additional Parameters of K2-266 from EXOFASTv2

\begin{tabular}{|c|c|c|c|c|}
\hline \multicolumn{4}{|c|}{ Wavelength Parameters: } & \multirow{2}{*}{$\begin{array}{l}\text { Kepler } \\
0.630_{-0.10}^{+0.064}\end{array}$} \\
\hline$u_{1}$ & & \multicolumn{2}{|c|}{ Linear limb-darkening coeff } & \\
\hline$u_{2}$ & & \multicolumn{2}{|c|}{ Quadratic limb-darkening coeff } & $0.107_{-0.054}^{+0.082}$ \\
\hline Telescope & & \multirow{2}{*}{\multicolumn{2}{|c|}{ Relative RV offset $\left(\mathrm{m} \mathrm{s}^{-1}\right)$}} & TRES \\
\hline$\gamma_{\text {rel }}$ & & & & $-29.9_{-8.6}^{+7.4}$ \\
\hline$\sigma_{J}$ & & \multicolumn{2}{|l|}{ RVjitter $\left(\mathrm{m} \mathrm{s}^{-1}\right)$} & $0.00_{-0.00}^{+19}$ \\
\hline$\sigma_{J}^{2}$ & & \multicolumn{2}{|l|}{ RV jitter variance } & $-130_{-140}^{+480}$ \\
\hline \multicolumn{5}{|c|}{ Transit Parameters: } \\
\hline \multirow[t]{2}{*}{ Planet } & Transit Date & \multirow{2}{*}{$\begin{array}{r}\text { Added Variance } \\
\sigma^{2} \times 10^{-10}\end{array}$} & Transit Mid Time & \multirow{2}{*}{$\begin{array}{l}\text { Baseline flux } \\
F_{0} \\
1.0000035 \pm 0.0000030\end{array}$} \\
\hline & Full $K 2 \mathrm{LC}$ & & $\begin{array}{l}\text { BJD }_{\mathrm{TDB}} \\
\mathrm{N} / \mathrm{A}\end{array}$ & \\
\hline$\overline{\mathrm{e}}$ & K2 UT 2017-06-10 & $19_{-18}^{+26}$ & $2457915.44761 \pm 0.00106$ & $0.999981 \pm 0.000016$ \\
\hline$f$ & K2 UT 2017-06-14 & $10_{-15}^{+21}$ & $2457919.05628 \pm 0.00088$ & $1.000012 \pm 0.000014$ \\
\hline $\mathrm{e}$ & K2 UT 2017-06-25 & $-3_{-11}^{+15}$ & $2457930.13813 \pm 0.00101$ & $1.000002_{-0.000012}^{+0.000013}$ \\
\hline $\mathrm{f}$ & K2 UT 2017-07-04 & $25_{-18}^{+26}$ & $2457938.54211 \pm 0.00108$ & $0.999986_{-0.000017}^{+0.0000016}$ \\
\hline $\mathrm{e}$ & K2 UT 2017-07-10 & $16_{-17}^{+25}$ & $2457944.83597 \pm 0.00137$ & $0.999971_{-0.000015}^{+0.000016}$ \\
\hline f & K2 UT 2017-07-23 & $54_{-27}^{+41}$ & $2457958.03343 \pm 0.00112$ & $0.999986_{-0.000021}^{+0.000020}$ \\
\hline $\mathrm{e}$ & K2 UT 2017-07-25 & $13_{-14}^{+21}$ & $2457959.52590 \pm 0.00090$ & $1.000000_{-0.000015}^{+0.000014}$ \\
\hline e & K2 UT 2017-08-08 & $30_{-19}^{+26}$ & $2457974.23919 \pm 0.00114$ & $1.000009 \pm 0.000017$ \\
\hline $\mathrm{f}$ & K2 UT 2017-08-12 & $5_{-15}^{+23}$ & $2457977.50614 \pm 0.00120$ & $1.000007 \pm 0.000015$ \\
\hline
\end{tabular}




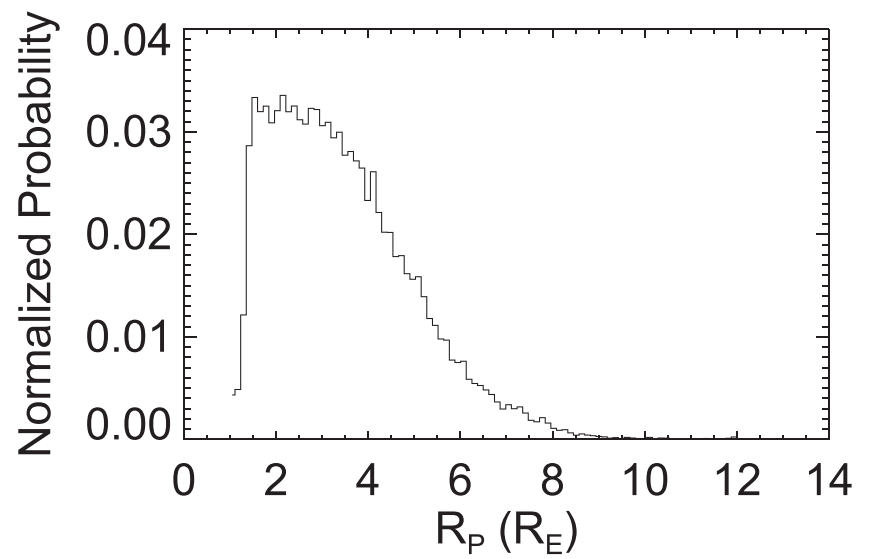

Figure 6. The probability distribution function for the radius of planet $b$. It shows that the depth of the transit sets a hard lower limit of $\sim 1 R_{\oplus}$. Due to the grazing geometry, the upper envelope is not constrained by the light curve, and instead is set by the upper limit on the mass through an RV non-detection and the Chen \& Kipping (2017) exoplanet mass-radius relation.

uncertainties to be Gaussian-distributed about the median transit time determined by EXOFASTv2, with variances set to the larger of the two asymmetric error bars in Table 5. Our likelihood function is then computed based on the standard chisquared statistic as $\ln \mathcal{L}=-\frac{1}{2} \chi^{2}$. We impose a Gaussian prior with 0 mean and a variance of $\sigma=0.05$ on the eccentricity vector components, $e_{i} \cos \omega_{i}$ and $e_{i} \sin \omega_{i}$, typical for eccentricities of multi-planet, sub-Neptune systems (Hadden \& Lithwick 2014, 2017; Van Eylen \& Albrecht 2015; Xie et al. 2016) We found that the inferred planet masses are insensitive to the assumed eccentricity priors after running additional MCMC fits with $\sigma=0.025$ and $\sigma=0.1$. We initialize our MCMC with 200 walkers and run for 120,000 iterations, saving all walker positions every 1000 iterations.

Figure 7 shows the observed timing variations of planets $d$ and e, along with $N$-body solutions drawn from our MCMC posterior. From our TTV dynamical fit, we determine planet-star mass ratios of $m_{d} / M_{*}=3.9_{-1.7}^{+2.5} \times 10^{-5}$ for planet $\mathrm{d}$ and $m_{e} / M_{*}=$ $6.3_{-2.2}^{+2.8} \times 10^{-5}$ for planet e. The TTVs yield no strong constraint on planet eccentricities and the posterior distributions largely mirror our assumed priors. We convert the dynamical constraints on planet-star mass ratios to constraints on the planetary masses and densities by combining the posterior samples from our TTV fit with posterior samples of our fit to stellar mass and planet radii computed with EXOFASTv2. The resulting posterior distributions of the planets' masses and densities are plotted in Figure 8. The inferred median and $1 \sigma$ planet mass values are $m_{d}=8.4_{-3.6}^{+5.4} M_{\oplus}$ and $m_{e}=13.6_{-4.7}^{+6.1} M_{\oplus}$, and the densities are $\rho_{d}=2.7_{-1.2}^{+1.8} \mathrm{~g} / \mathrm{cm}^{3}$ and $\rho_{e}=5.6_{-2.0}^{+2.6} \mathrm{~g} / \mathrm{cm}^{3}$.

Our $N$-body dynamical model contains 10 free parameters, which are fit to only nine data points. This means that, at face value, our model is underconstrained and we are at risk of overfitting. To understand the origin of our dynamical mass constraints and ensure that they are not merely the result of overfitting, we analyze the TTVs using the analytic model of Hadden \& Lithwick (2016). This analytic treatment reduces the dimensionality of the TTV model so that it is no longer underconstrained. Note that we adopt the masses derived from the more complete $N$-body model as our best-fit values; we present the analytic model simply as a consistency check to
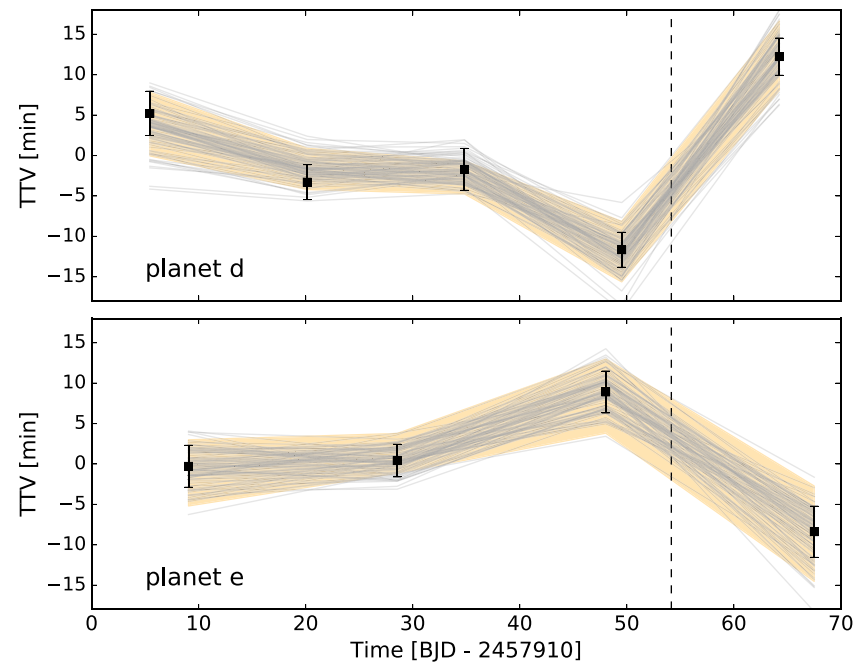

Figure 7. The observed transit timing variations of planet's d and e are shown by black squares with error bars showing the $1 \sigma$ uncertainties. A sample of 100 $N$-body solutions drawn from our MCMC fit posteriors are shown in gray. The shaded orange region shows the $1 \sigma$ uncertainty in the analytic TTV model fit via least squares. The time at which $\mathrm{d}$ and e experience a conjunction is indicated by the dashed vertical.

ensure that the $N$-body results are not overfitting the data because of poor MCMC convergence.

We use the formulas of Hadden \& Lithwick (2016) to construct an analytic model for the TTVs of planets $d$ and e as a function of planet periods $P_{i}$, initial transit times $T_{i}$, planet-star mass ratios $\mu_{i}$, and the complex "combined eccentricity"

$$
\mathcal{Z} \approx \frac{1}{\sqrt{2}}\left(e_{e} e^{i \omega_{e}}-e_{d} e^{i \omega_{d}}\right) .
$$

The analytic model reduces the total number of model parameters to eight by combining the planets' eccentricities and longitudes of perihelia into the single complex quantity, $\mathcal{Z}$. The $n$th transit of planet $\mathrm{d}$ and e are modeled as

$$
t_{i}(n)=T_{i}+n P_{i}+\delta t_{\mathcal{C}, i}(n)+\delta t_{\mathcal{F}, i}(n)
$$

where $\delta t_{\mathcal{C}} \propto \mu^{\prime}, \delta t_{\mathcal{F}} \propto \mu^{\prime} \mathcal{Z}$ with $\mu^{\prime}$ being the perturbing planet's planet-star mass ratio. Expressions for $\delta t_{\mathcal{C}}$ and $\delta t_{\mathcal{F}}$ are given in Hadden \& Lithwick (2016). We use the Levenberg-Marquardt minimization algorithm to fit our analytic model to the observed transit times and estimate uncertainties from the local curvature of the $\chi^{2}$ surface (e.g., Press et al. 1992). The analytic fit, plotted in Figure 7, yields masses $m_{d} / M_{*}=3.0 \pm 0.8 \times 10^{-5}$ for planet $\mathrm{d}$ and $m_{e} / M_{*}=4.6 \pm 1.0 \times 10^{-5}$ for planet e, which are consistent with the $N$-body MCMC constraints.

The origins of the mass constraint can be qualitatively understood from the analytic model as follows: at conjunction, planets impart impulsive kicks to one another that change their instantaneous orbital periods. This effect is captured by the socalled "chopping" terms, $\delta t_{\mathcal{C}}$, in Equation (2) (see also Nesvorný \& Vokrouhlický 2014; Deck \& Agol 2015). Indeed, we obtain nearly identical mass constraints from an analytic fit that drops the $\delta t_{\mathcal{F}}$ terms from Equation (2) (and thereby further reduces the number of free parameters to six as the model no longer depends on the complex number $\mathcal{Z}$ ). Because these $\delta t_{\mathcal{F}}$ terms vary over a timescale much longer than the baseline of our observations, they are well-approximated by a linear trend and essentially degenerate with small changes to the $T_{i}+n P_{i}$ 

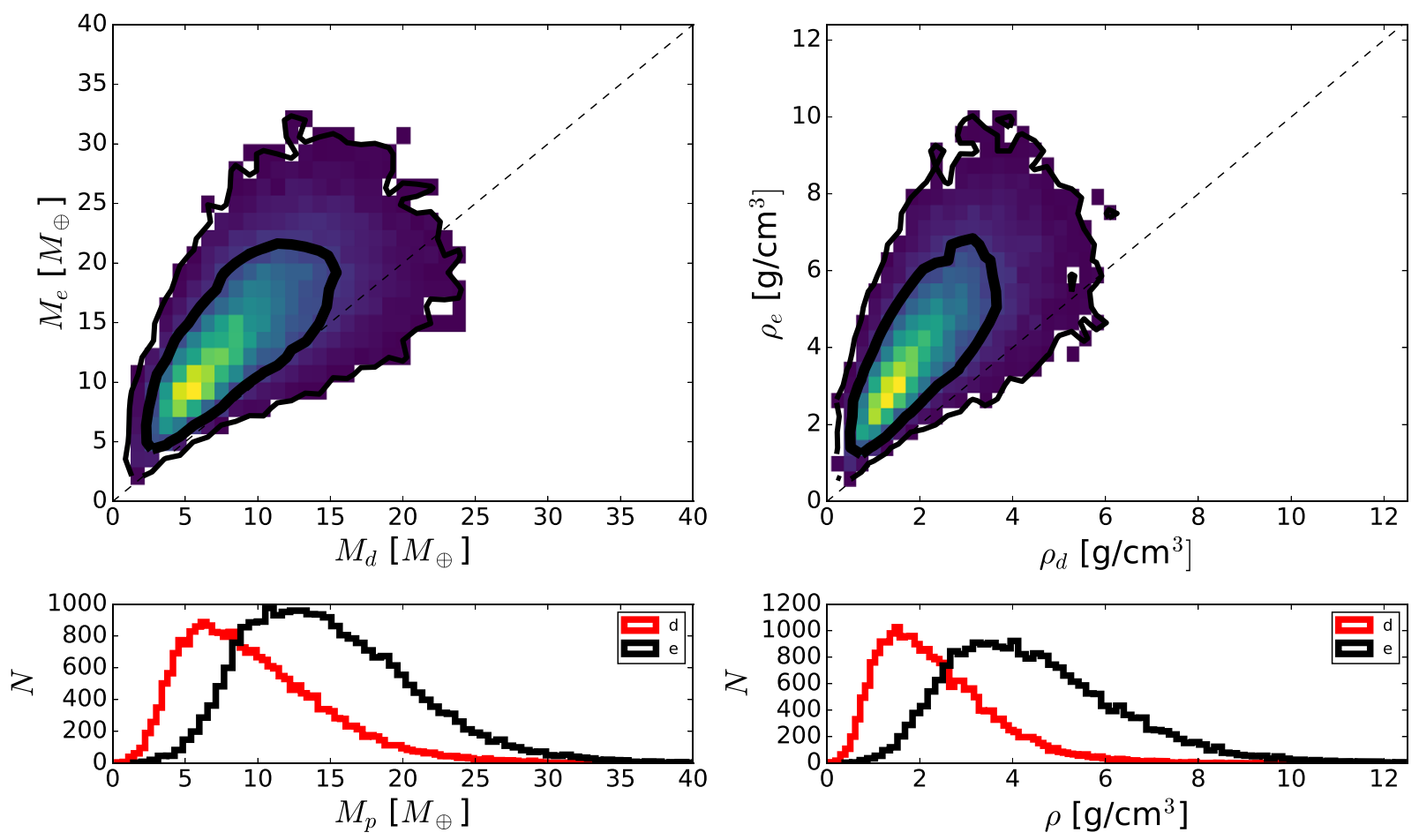

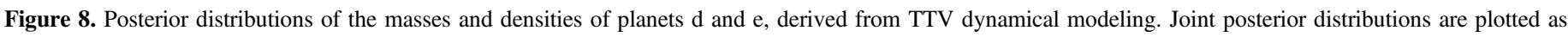

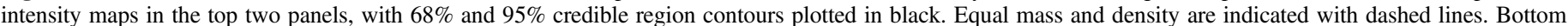
panels show one-dimensional histograms of the marginalized posterior distribution of the mass and density of planets d (red) and e (black).

terms in Equation (2). Thus, we have identified the the origin of our mass constraints with the measurement of the chopping signals, $\delta t_{\mathcal{C}, i}$, in the TTVs of planets $\mathrm{d}$ and e. Over the course of our observing baseline, planets $d$ and e experience a single conjunction, at the time marked by a dashed line in Figure 7. The power of the TTV signal for constraining the planets' masses comes mainly from the impulsive changes in the planets' osculating orbital periods experienced at this conjunction causing the planets to arrive early (in the case of e) or late (in the case of $d$ ) at their next transits.

\subsection{Dynamical Stability and Transit Likelihood}

Next, we consider the dynamical stability of the system, along with the probability that all of the putative planets can be seen in transit. Although most Kepler multi-planet systems tend to have low mutual inclinations, this system is unique to date because there is a significant mutual inclination between the innermost planet and the other five. In the context of the known set of multi-planet systems, this appears significant. Ballard \& Johnson (2016) found that the Kepler planet-hosting systems around cool stars appears to be drawn from two populations: one set of multi-transit systems and a second set of singletransit planets, which may also have statistically higher obliquities (Morton \& Winn 2014) (the concept of these two populations is commonly called the "Kepler dichotomy"). One solution to the Kepler dichotomy is that the two populations are actually all multi-planet systems, and that the ones that appear to be singly-transiting are really systems with larger mutual inclinations, which can see seen as single-transit systems from a particular line of sight. Although most Kepler multi-planet systems (those with four or more planets) are fairly tightly confined to a roughly coplanar region, there is some precedent for multi-planet systems: Mills \& Fabrycky (2017) found a two-planet system with a $24^{\circ}$ mutual inclination. In cases like this, the question of how many planets in a multi-transiting system might be seen in transit at any one time becomes relevant (Brakensiek \& Ragozzine 2016), as large mutual inclinations can lead to only a subset of the planets being seen from a given line of sight. K2-266 b is currently observed to have a grazing transit and a high mutual inclination with the remainder of the planets, the five of which reside in a roughly coplanar configuration. With an aim toward assessing where this system fits into the Kepler dichotomy, in this section we conduct an analysis of the transit likelihood for various numbers of planets in this system.

To test the dynamical and transit stability of these planets, we ran $250 \mathrm{~N}$-body simulations of the system, drawing the initial orbital elements from the posteriors generated from the EXOFAST transit fit (more specifically, we draw a single link from the MCMC posterior at random for each of the 250 simulations, and then use all orbital elements from that link). We assigned the longitude of ascending node to be $2 \pi$, as it cannot be measured from the transit fits. The planetary masses are drawn from the posteriors provided by the EXOFAST fit, as are stellar mass and radius. For all calculated values of inclination, we broke the above/below solar midplane degeneracy by randomly assigning the value to be either greater or smaller than $90^{\circ}$. We also assume that the stellar obliquity is aligned with the plane containing the outer five planets (but there are no system-specific observations to support this assumption; instead, we make this assumption as a computational necessity, although we expect the stellar obliquity to be more aligned in multi-planet systems; see Morton \& Winn (2014)). These 250 simulations were carried out using the hybrid Wisdom-Holman and Bulirsch-Stoer (BS) integrator Mercury6 (Chambers 1999) for integration times of $10^{5}$ years, and with an initial time step of 8.5 minutes. 


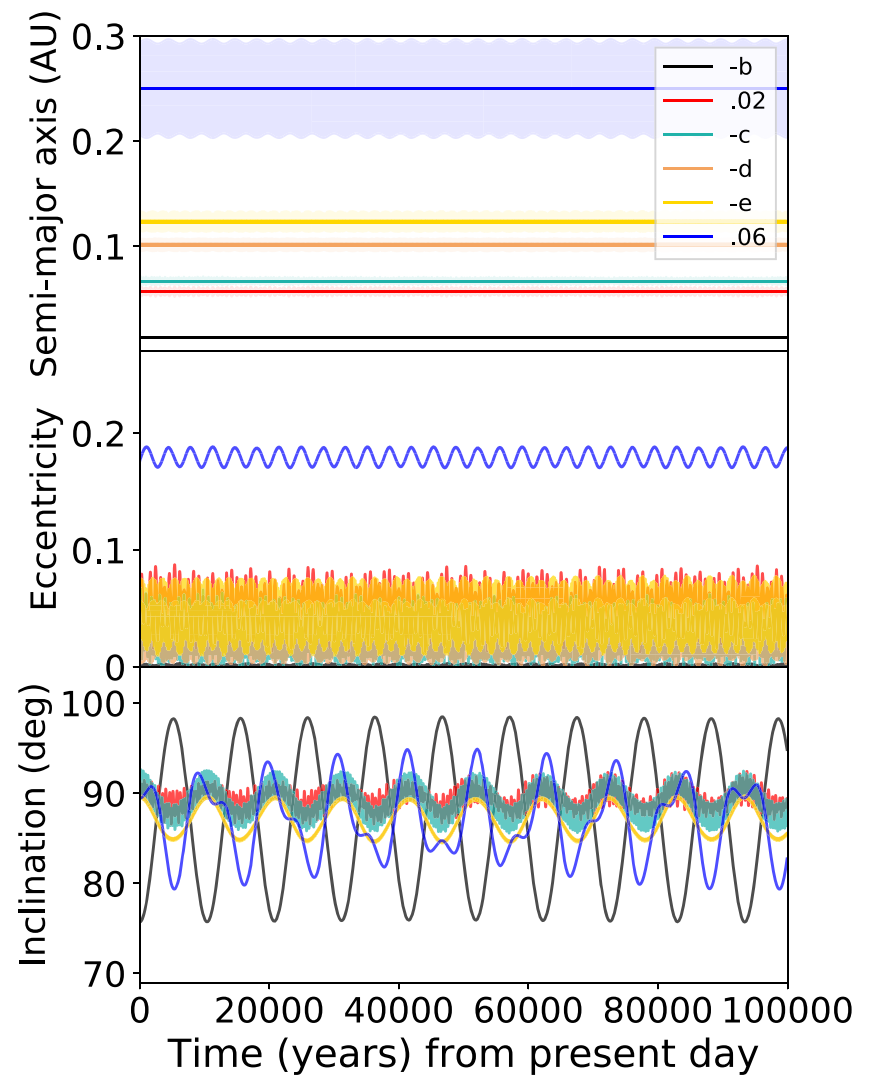

Figure 9. A sample integration from the suite of 250 run for this work, plotted for illustrative purposes. This is a typical dynamically stable integration, where planetary orbits do not wander far from their initial values of semimajor axis or eccentricity. Top panel: the semimajor axis of each planet, with shaded regions to denote the extent of the perihelion and aphelion distances. Middle panel: the eccentricity evolution of each planet, which oscillate but remain confined near their initial values. Bottom panel: inclination values for each planet. The inclination values for all planets oscillate with varying amplitudes, as is typical for all integrations.

Energy was conserved to better than one part in $10^{8}$ over the course of the simulations. When physical collisions occur, particles are removed from the simulation. The integration time was chosen to include many secular timescales of the system (Figure 9 shows that many periods of secular oscillations are included in the $10^{5}$ years time span).

In roughly $66 \%$ of our suite of 250 simulations, at least two planets in the system attain orbits that cross. In $23 \%$ of the simulations, the system experiences a true dynamical instability, in which a planet is ejected from the system or physically collides with another body. In the cases in which orbits cross, planets 0.02 and $\mathrm{c}$ are the culprits of the instability roughly $80 \%$ of the time. On the $10^{5}$ year integrations considered in this work, a sizable fraction (roughly a third) remain dynamically stable. As such, we cannot use dynamical arguments to argue against the existence of planet candidate 0.02 , whose close orbit with planet c might otherwise be suspect.

As neither of the planet candidates can clearly be ruled out based on dynamical arguments, we next consider the dynamical evolution that leads to only a subset of the six known planets being seen in transit. We currently observe the system to be a six-planet system, but the innermost planet K2-266 b has a high measured impact parameter, meaning that is barely transiting. The simulations show significant inclination evolution over time for both K2-266 b and the other planets in the system. In

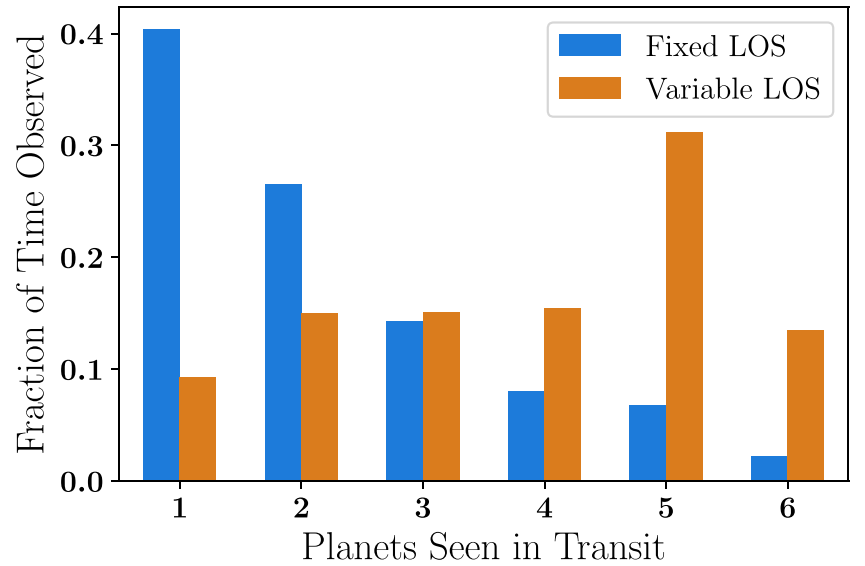

Figure 10. The future simultaneous transit fraction by the number of planets seen in transit. These fractions were computed using the numerical simulations for two lines of sight: a fixed line of sight (Fixed LOS), set to be the current line of sight from the earth, and a variable line of sight (Variable LOS), computed at each time step to be the line of sight from which the greatest number of planets can be seen in transit at any time. The simulations used to construct this plot are the subset of the 250 simulations run in this section. Given the measured orbital elements of the planets around K2-266, the system is expected to be observed as a six-planet system a minority of the time (assuming there are no extra unseen planets in the system).

Figure 9, we plot a representative case from our set of 250 simulations, where the semimajor axes and eccentricities of all planets remain confined relatively close to their currently measured values, but the orbital inclination of all six planets evolves.

One notable feature of the numerical simulations is the inclination evolution of all six planets. Secular evolution of the system causes the planetary orbits to evolve with time, resulting in configurations in which not all planets can be simultaneously seen in transit. Inspired by the present-day (apparently serendipitous) geometry, we extracted from the simulations the transit probability over time for varying numbers of planets in this system. The result of this analysis is presented in Figure 10 for two lines of sight. The first case considers the fixed line of sight corresponding to our current location (that of the solar system). The second case uses an optimized variable line of sight, which is recomputed at each time step of each integration to determine the largest multiplicity that can be observed from any location in the galaxy.

This analysis shows that observing six transiting planets in the system is rare, given the known components of the system. No matter which line of sight is considered, the system will appear to contain the six "known" planets a minority of the time. More commonly, the system will be seen as a five-planet system from the most favorable line of sight, and as a one- or two-planet system from our current line of sight. Most of the time, the inclinations of the outer five planets evolve and cause them to reside in non-transiting configurations. On the other hand, the probabilities are not vanishingly small. We expect to be able to reobserve a six-planet transiting system about $2.2 \%$ of the time in the future from our current line of sight, given the currently measured orbits of these planets. It is also important to note that we cannot be sure that the observed six planets are the only planets in this system: additional, non-transiting planets would alter the dynamics described here. 


\subsection{Resonant State of the Two Sub-Neptunes}

The sub-Neptunes K2-266 d and e, with periods of 19.482 days and 14.697 days, have a period ratio of 1.326 , which is $0.59 \%$ away from the $4: 3$ mean motion commensurability. These planets reside in nearly the same orbital plane (with $89^{\circ} .73$ and $89^{\circ} .74$ inclinations). As such, the orbital periods of these planets suggest that they may reside in orbital resonance. However, true resonance is characterized by a librating resonance angle, and it is not clear from the orbital elements alone whether the resonance angle will librate or circulate. To determine the true resonance behavior of these two planets, we used the subset of the 250 simulations that did not experience orbit-crossing during the $10^{5}$ year integration time, and postprocessed the results to search for resonances.

We have performed a resonance-finding algorithm to identify the time intervals in the simulations where the planets were in true resonance. We found regimes with nearly constant period ratio (constant $P_{e} / P_{d}$ ), generated arrays of resonance angles for all $p: q$ resonances up to 29th order (while $p \leqslant 30$ ), and automatically generated plots using the simulated orbital elements of planets K2-266 d and e for each resonance angle for each resonance order for all of the 250 simulations. Using the resulting resonance angles, we searched for librating behavior by breaking the time series into 5000 year intervals and searching for gaps in resonance angle space: Note that a circulating resonance angle will populate the entire $360^{\circ}$ range of possible angles, whereas a librating angle will have gaps.

We find that, in our simulations, planets K2-266 d and e exhibit orbital resonances approximately $8.1 \%$ of the time over the simulations under consideration. The resonance angles populated in these cases have the forms

$$
\psi_{1}=4 \lambda_{o}-3 \lambda_{i}-\varpi_{o}
$$

and

$$
\psi_{2}=4 \lambda_{o}-3 \lambda_{i}-\varpi_{i}
$$

where $\lambda$ is mean longitude and $\varpi$ is longitude of pericenter. The subscripts denote the inner $(i)$ and outer $(o)$ planets. The four types of resonance behavior exhibited by this system, in order of occurrence rate, include: non-resonance, continuous resonance for the entire simulation lifetime, an initial condition close to resonance that loses the resonance as the system evolves, and very rarely, the attainment of a resonance after an initial period of non-resonance (see Ketchum et al. (2013) for a more detailed discussion of this process). We find that, for trials that start out in a resonant configuration, the typical libration width of the resonance generally is consistently around $190^{\circ}$. Although this width may evolve slightly as the simulation progresses, the resonances are not typically much deeper than this initial value.

\subsection{Chaotic Behavior}

Dynamical systems are often chaotic and we would like to quantify the chaotic behavior of K2-266. The system, as observed, has six planets in a compact configuration with a relatively large mutual inclination between the innermost planet and the others. Our numerical simulations, described above, indicate that while the outer planets (K2-266 d, e, and .06) are generally dynamically stable, the middle planets (K2-266.02 and d) can experience scattering or other non-periodic time evolution, potentially leading to orbit crossing. Non-periodic behavior of this nature can be indicative of chaos.

The evolution of a chaotic planetary system is extremely sensitive to its initial conditions. Chaos is often parameterized by the Lyapunov exponents of the system, which determine the rate of exponential divergence of orbits with similar initial conditions. In contrast, general observational uncertainties in the orbital elements can also lead to non-chaotic divergence if initial orbital elements are drawn from different locations of the posteriors (in cases where ensembles of simulations are used to sample the uncertainties). Either sufficiently large observational errors or the sufficiently rapid onset of chaos will thus make both numerical integrations and analytic explorations less certain.

To test the chaotic behavior of the K2-266 system, we ran 400 integrations of this system, with the orbital parameters and masses drawn from the posteriors generated by the transit fit. Each simulation was carried out using the Rebound N-Body integration package (Rein \& Liu 2012), where we used a total integration time of 1000 years, the IAS15 integrator (Rein \& Tamayo 2015), and an initial time step of eight minutes. For each of these integrations, we evaluate the chaotic nature of the initial conditions by employing the Mean Exponential Growth factor of Nearby Orbits (MEGNO) indicator (Cincotta et al. 2003), implemented in the Rebound N-body code. For chaotic trajectories, the MEGNO indicator, $Y$, grows linearly in time at a rate of $1 / t_{L y}$ where $t_{L y}$ is the Lyapunov time, while for regular trajectories it asymptotically approaches $Y=2$. We compute MEGNO values for the 400 draws from the posteriors of this system at times between 1 and 1000 years. These realizations provide a good sample of the parameter space spanned by the observational posteriors. Of the 400 realizations, only $4.5 \%$ can be categorized as unambiguously regular at the end of the integration (where the criterion for regularity is taken to be MEGNO < 4). Moreover, we find no strong correlations between planetary parameters and MEGNO values using our current simulation set. We attempted to trace chaotic behavior using the period ratio of the resonant planets $\mathrm{d}$ and $\mathrm{e}$ (as done in Figure 3 of Deck et al. 2012), the ratio perihelion/ aphelion of .02 and c, and by using the orbital elements of planet $b$, but no trends emerged. This finding is likely due to the high multiplicity and tightly packed nature of the system: there are multiple equally-important sources of dynamical chaos. In Figure 11, we plot the median MEGNO indicator value for these 400 simulations considered in this section at periodic intervals in the 1000 year integrations. A MEGNO indicator less than four denotes regular orbits, while a MEGNO of four or greater denotes measured chaos. The median MEGNO indicator reaches four (denoting the measurable onset of chaos) at roughly 100 years.

From this MEGNO analysis, we know that the majority of orbits allowed by the transit posteriors are chaotic. It is important to note that, while some of these chaotic posterior points are likely destined to experience instabilities based on our $10^{5}$ year numerical simulations, a chaotic system does not necessarily mean a dynamically unstable system, or even a particularly active system (the planetary orbits in our solar system are known to be chaotic, as is the Kepler-36 planetary system; see Deck et al. (2012)). Chaos implies that similar initial conditions will diverge over some timescale, so precise future predictions of planetary orbits can no longer be made. 


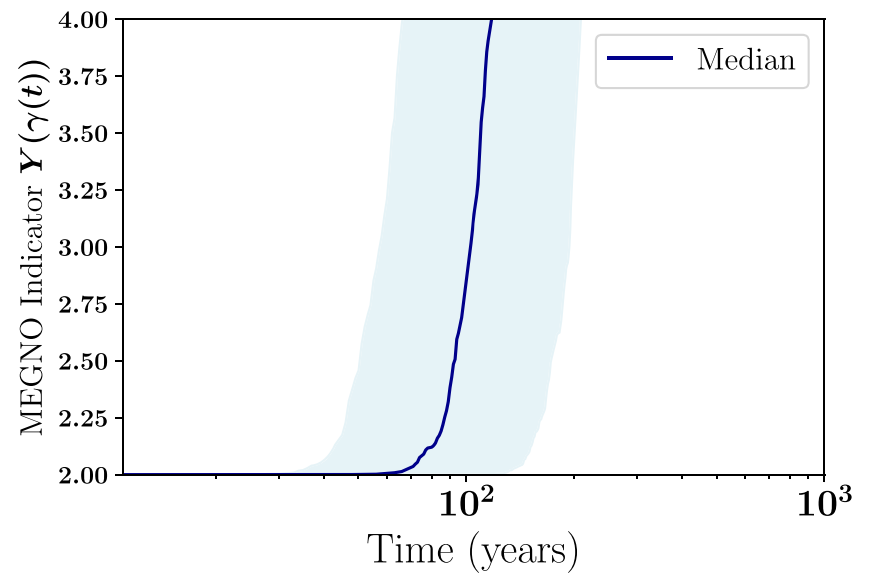

Figure 11. The median MEGNO indicator value as a function of time during the thousand-year Rebound integrations (among 400 realizations). The median value reaches $Y=4$, indicating chaotic behavior, after only 100 years. The light blue shaded region delineates the quartile values of the MEGNO indicator. For the majority of posterior draws, this system is thus highly chaotic.

Specifically, for two given sets of similar initial conditions, integrations of both cases could result in systems that are dynamically stable and continuously transit, but the values of the phase space variables (including planet locations) can diverge over time if the system is chaotic. One implication of this analysis is that a large amount of uncertainty in forward integrations comes from chaos, rather than only from the uncertainty of the transit posteriors.

\section{EPIC 248435395: a Likely Co-moving Companion with a Transiting Planet Candidate}

After the identification of the six possible planets orbiting K2-266, we searched for nearby stellar companions to understand the full architecture of the system, because that may help disentangle the origin of the misaligned inner planet. About $\sim 42^{\prime \prime}$ from K2-266 is EPIC 248435395, which is $\sim 1.7 \mathrm{mag}$ fainter in the $V$ band ( $~ 0.6$ mag fainter in the $K$ band).

\subsection{Evidence for Companionship}

To check that EPIC 248435395 is actually an associated companion to K2-266, we directly compare the Gaia Data Release 2 proper motions and distances for both systems (Gaia Collaboration et al. 2016, 2018). K2-266 has a Gaia parallax of $12.87 \pm 0.06$ mas, corresponding to a distance of $77.8 \pm 0.6 \mathrm{pc}$, while EPIC 248435395 has a parallax of $12.85 \pm 0.06$ mas and a distance of $77.7 \pm 0.6 \mathrm{pc}$. All systematic uncertainties on the Gaia DR2 parallax should be $<0.1$ mas. Therefore, the two stars are at the same distance. The Gaia DR2 proper motions for K2266 are $\mu_{\alpha}=56.9$ mas yr $^{-1}$ and $\mu_{\delta}=-68.8$ mas yr $^{-1}$. These are very similar to the proper motions for EPIC 248435395,

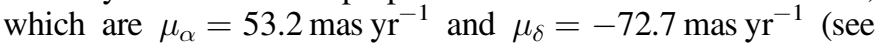
Table 1). The Gaia parallaxes and proper motions are consistent with the two stars being a widely separated binary.

Using this distance, the $\sim 42^{\prime \prime}$ separation would correspond to an orbital semimajor axis of $\sim 3200$ au. Using our determined mass of EPIC 248435395 of $0.561 M_{\odot}$ and K2-266 of 0.649 $M_{\odot}$ this would correspond to an orbital period 0.16 Myr. This period would result in a $\sim 4$ mas motion from the binary orbit, within the expected micro-arcsecond expected astrometric precision (Gaia Collaboration et al. 2018). However, EPIC 248435395 will move $\sim 0$ ! 45 over the nominal five-year Gaia mission, and it may be difficult to differentiate the contribution from the binary orbit from the star's proper motion. Moreover, as we show in the following section, EPIC 248435395 is likely a binary itself, which could further confuse the astrometric solution.

\subsection{Observations of EPIC 248435395}

The $\sim 42^{\prime \prime}$ separation means that the two systems were wellresolved by $K 2$. We were therefore able to produce a separate light curve for the companion star. As we did for K2-266 and following the strategy described in Vanderburg \& Johnson (2014) and Vanderburg et al. (2016a), we searched EPIC 248435395 for possible transit signals. From this search, we identify a transit signal at 8.5 days with an $\mathrm{S} / \mathrm{N}$ of 7.0 around EPIC 248435395, below the typical Kepler $\mathrm{S} / \mathrm{N}$ threshold of 7.1 and after $K 2$ of 9 . The final light curve for EPIC 248435395 has a 30 minute noise level of $151 \mathrm{ppm}$ and a 6 hour photometric precision of $42 \mathrm{ppm}$.

We also inspected the NGS POSS and Pan-Stars images of EPIC 248435395 in a manner similar to that described in Section 2.4 (see Figure 3). Because it shares a common proper motion with K2-266, it has also moved about 6 arcseconds since it was imaged by POSS in 1952. Because EPIC 248435395 is fainter than K2-266, its saturated point spread function does not extend all the way to the star's current-day position, so we are able to rule out background objects down to the POSS limiting magnitude of about 20 in the red, and 19 in the blue. We identified no nearby companions in Pan-STARRS imaging. We also obtained high-resolution images in the $\mathrm{Br}-\gamma$ filter and the $J$ band of EPIC 248435395 using NIRC2 on UT 2017 December 28 (see Section 2.5 and Figure 3). We see no evidence for any additional companions from our "patient" and AO imaging.

We also obtained two spectra of EPIC 248435395 using TRES, on UT 2018 February 7 and UT 2018 April 3. They were analyzed according to the same procedures described in Section 2.2. The absolute RV of EPIC 248435395 is $12.84 \pm 0.31 \mathrm{~km} \mathrm{~s}^{-1}$, about $2 \mathrm{~km} \mathrm{~s}^{-1}$ different from K2-266. Given their projected separation, this is slightly larger than one would expect if the two stars are bound. However, we note that this is unlikely to be the true systemic velocity of EPIC 248435395. The large error on the velocity is not because of poor RV precision, but because our two RVs exhibit a 450 $\mathrm{m} \mathrm{s}^{-1}$ variation; EPIC 248435395 is likely to be a binary itself, and we do not know the full amplitude of variation or its orbital phase. We conclude that the RVs of the components of the wide pair are not inconsistent with expectations for a bound system; K2-266 is likely to be the primary star in a hierarchical triple system. The TRES RVs for EPIC 248435395 are shown in Table 2.

\subsection{EXOFASTv2 Global Fit for EPIC 248435395}

Following a procedure similar to the one used for K2-266 (see Section 3), we perform a fit of the exoplanet candidate around EPIC 248435395 using EXOFASTv2 (Eastman 2017). Within this analysis, we simultaneously fit the flattened $K 2$ 

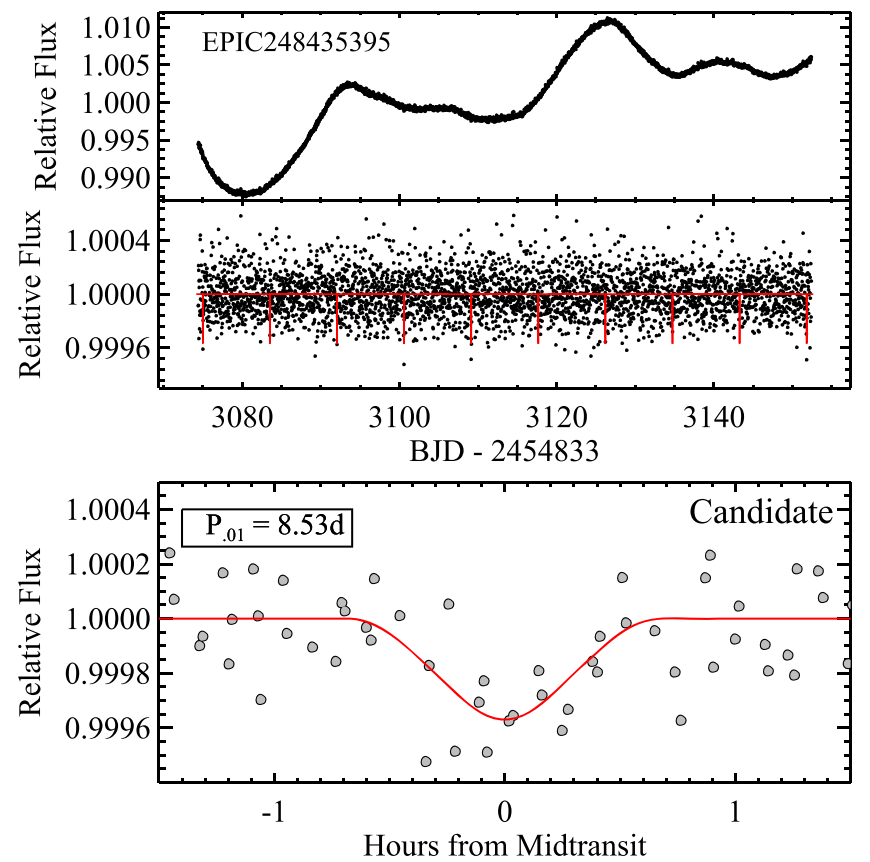

Figure 12. Top: the full $K 2$ light curve of EPIC 248435395 from Campaign 14, corrected for systematics using the technique described in Vanderburg \& Johnson (2014) and Vanderburg et al. (2016a). The observations are plotted in open black circles. Middle: the flattened final $K 2$ light curve used in the EXOFASTv2 fit. Bottom: the corrected $K 2$ light curve phase-folded to the 8.53 day period identified for the planet candidate around EPIC 248435395. The red line corresponds to the final best-fit EXOFASTv2 transit model.

light curve (see Figure 12) and SED (see Figure 4) for EPIC 248435395. To constrain the mass of EPIC 248435395 within the fit, we use a Gaussian prior of $0.584 \pm 0.015 M_{\odot}$ from Mann et al. (2015), but with the uncertainties inflated to $5 \%$. We constrain the radius within the fit using the broadband photometry shown in Table 1 . We set a starting point on the $T_{\text {eff }}$ and $[\mathrm{Fe} / \mathrm{H}]$ of the host star to be $3699 \mathrm{~K}$ and -0.006 dex from the EPIC catalog (Huber et al. 2016). We also enforce the same upper limit on the $V$-band extinction from the Schlegel et al. (1998) dust maps of 0.0548 as we did for the fit of K2-266. We use the Gaia DR2 parallax (12.85 mas) with a conservative uncertainty of 0.1 mas as prior in the fit. The final determined system parameters are shown in Tables 3 and 7 . We note that this is not a confirmed or validated planet.

\section{Discussion}

The complex architecture of the planetary system surrounding K2-266 makes it an intriguing target for further characterization. Additionally, the host star is relatively bright $(V=11.8, K=8.9)$ and up to six planets orbit the host star in a compact configuration. At the present time, we are only able to validate planets $\mathrm{b}, \mathrm{c}, \mathrm{d}$, and e, so more data are needed to confirm the remaining two candidates.

\subsection{Atmospheric Characterization}

The origin of Neptune-sized planets is not clear, yet they appear to be one of the most common types of planets. The planets range in size from $2-6 R_{\oplus}$ and have been discovered orbiting $>25 \%$ of all stars (Howard et al. 2012; Fressin et al. 2013; Buchhave et al. 2014; Fulton et al. 2017). Recent statistical studies of the observed amplitude of transmission spectral features of warm Neptunes show a correlation with equilibrium temperature or its bulk $\mathrm{H} / \mathrm{He}$ mass fraction (Crossfield \& Kreidberg 2017). However, there are only a small number of Neptune-sized planets that are amenable to transmission spectroscopy with current facilities like the Hubble Space Telescope (HST).

To understand if the planets orbiting K2-266 would be viable targets for transmission spectroscopic measurements, we follow the technique described in Vanderburg et al. (2016a) to calculate their expected atmospheric scale height and $\mathrm{S} / \mathrm{N}$ per transit. Using data from NASA's Exoplanet archive (Akeson et al. 2013), we also calculate the atmospheric scale height and $\mathrm{S} / \mathrm{N}$ for all planets with $R_{p}<3 R_{\oplus}$ (see Table 6), updating this table from Rodriguez et al. (2018). The calculations are done in the $H$-band to understand their accessibility using the Wide Field Camera 3 instrument on $H S T$, as well as the future feasibility to observe them with the suite of instruments on the upcoming James Webb Space Telescope (JWST). Purely based on the inferred sizes of the planets, it is expected that K2-266 b, d, and e might all have thick gaseous atmospheres (Weiss \& Marcy 2014), but the uncertainty in the size of planet b (due to the grazing transit configuration) and its proximity to its host star makes this unclear (see Figure 6 for the probability distribution of planet b's radius from our global fit). While our transit fit indicates that the most probable size of planet $b$ is about three times the size of the Earth, virtually all known USP planets known are smaller than $2 R_{\oplus}$ (Sanchis-Ojeda et al. 2014; Winn et al. 2017)

$\mathrm{K} 2-266 \mathrm{~b}$ is a particularly interesting target for atmospheric follow-up because of its status as a USP exoplanet. Lopez (2017) suggests that, in order for USP planets to have radii larger than $\sim 2 R_{\oplus}$, they should have formed with highmetallicity, water-rich envelopes, and are likely to remain water-rich today. In addition, the theoretical models of Owen \& $\mathrm{Wu}$ (2013) and Owen \& Wu (2017) suggest that planets with the radial size and orbital period of K2-266 b reside near the boundary in parameter space where photoevaporation becomes an important source of mass loss. In addition, if K2-266 b has a typical magnetic field strength, its close proximity allows for interactions between the magnetospheres of the star and the planet (Adams 2011). Combinations of mass measurements of $\mathrm{K} 2-266 \mathrm{~b}$, refined radius measurements, and atmospheric constraints on water abundance could be used together to paint a complete picture of where in the disk this planet originated and when it reached its current-day location.

K2-266 d and e are two of the best sub-Neptunes for atmospheric characterization, and their longer periods (as compared to the others in Table 6) provide a valuable opportunity for a comparative atmospheric study between hot and warm sub-Neptunes. Interestingly, the Near-IR and IR brightness of K2-266 and EPIC 248435395 should allow for high-S/N observations using short exposure time for all four instruments on JWST: Near Infrared Camera (NIRCam), Near Infrared Imager and Slitless Spectrograph (NIRISS), NearInfrared Spectrograph (NIRSpec), and the Mid-Infrared Instrument (MIRI) (Beichman et al. 2014; Kalirai 2018). 
Table 6

The Best Confirmed or Validated Planets for Transmission Spectroscopy with $R_{P}<3 R_{\oplus}$

\begin{tabular}{|c|c|c|c|c|c|}
\hline Planet & Period (days) & $R_{P}\left(R_{\oplus}\right)$ & $\mathrm{S} / \mathrm{N}^{\mathrm{a}}$ & Reference & Discovery \\
\hline GJ 1214 b & 1.58 & 2.85 & 1.00 & Charbonneau et al. (2009) & MEarth \\
\hline $55 \mathrm{Cnc}$ e & 0.74 & 1.91 & 0.41 & Dawson \& Fabrycky (2010) & RVs \\
\hline HD 97658 b & 9.49 & 2.35 & 0.27 & Dragomir et al. (2013) & RVs \\
\hline TRAPPIST-1 f & 9.21 & 1.04 & 0.24 & Gillon et al. (2017) & Spitzer \\
\hline $\mathrm{K} 2-136 \mathrm{c}$ & 17.31 & 2.91 & 0.19 & Ciardi et al. (2018), Livingston et al. (2018), Mann et al. (2018) & $K 2$ \\
\hline GJ 9827 b & 1.21 & 1.75 & 0.18 & Niraula et al. (2017), Rodriguez et al. (2018) & $K 2$ \\
\hline $\mathrm{K} 2-167 \mathrm{~b}$ & 9.98 & 2.82 & 0.16 & Mayo et al. (2018) & $K 2$ \\
\hline $\mathrm{K} 2-266 \mathrm{e}$ & 14.70 & 2.93 & 0.15 & This work & $\mathbf{K} 2$ \\
\hline GJ 9827 d & 6.20 & 2.10 & 0.15 & Niraula et al. (2017), Rodriguez et al. (2018) & $K 2$ \\
\hline HIP $41378 \mathrm{~b}$ & 15.57 & 2.90 & 0.14 & Vanderburg et al. (2016a) & $K 2$ \\
\hline HD $3167 \mathrm{~b}$ & 0.96 & 1.70 & 0.14 & Vanderburg et al. (2016b), Christiansen et al. (2017) & $K 2$ \\
\hline $\mathrm{K} 2-233 \mathrm{~d}$ & 24.37 & 2.65 & 0.13 & David et al. (2018) & $K 2$ \\
\hline $\mathrm{K} 2-266 \mathrm{f}$ & 19.48 & 2.73 & 0.12 & This work & K2 \\
\hline $\mathrm{K} 2-28 \mathrm{~b}$ & 2.26 & 2.32 & 0.12 & Hirano et al. (2016) & $K 2$ \\
\hline K2-199 c & 7.37 & 2.84 & 0.12 & Mayo et al. (2018) & $K 2$ \\
\hline $\mathrm{K} 2-155 \mathrm{c}$ & 13.85 & 2.60 & 0.11 & Diez Alonso et al. (2018), Hirano et al. (2018) & $K 2$ \\
\hline Kepler-410 A b & 17.83 & 2.84 & 0.10 & Van Eylen et al. (2014) & Kepler \\
\hline HD $106315 \mathrm{~b}$ & 9.55 & 2.40 & 0.10 & Rodriguez et al. (2017), Crossfield et al. (2017) & $K 2$ \\
\hline
\end{tabular}

Note.

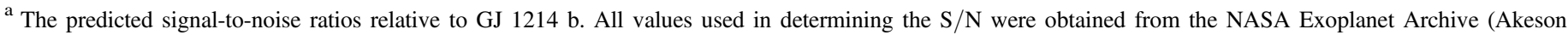

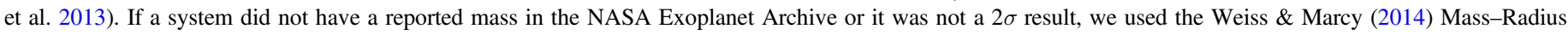
relationship to estimate the planet's mass.

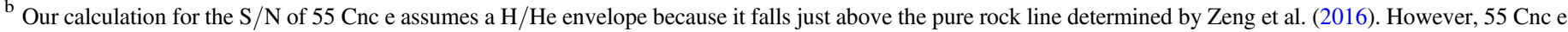
is in a USP orbit, making it unlikely that it would hold onto a thick $\mathrm{H} / \mathrm{He}$ envelope. We do not include K2-266 b due to its grazing configuration.

\subsection{Dynamical Classification}

The dynamics of the K2-266 system is characterized by several remarkable features: The innermost planet (K2-266 b) is highly inclined relative to the rest of the planets, the orbits of planet candidate K2-266.02 and validated planet K2-266 c are in close proximity, the two sub-Neptunes (K2-266 d, e) are either in or extremely close to a mean-motion resonance, and the outer candidate K2-266.06 has a moderately eccentric orbit. Taken together, these factors place the planetary system orbiting K2-266 in a particularly unique realm.

Among the exoplanetary systems discovered thus far, only a small number have been determined to be in true resonance (Rivera et al. 2010; Lissauer et al. 2011; Carter et al. 2012; Barclay et al. 2013; Mills et al. 2016; Luger et al. 2017; Millholland et al. 2018; Shallue \& Vanderburg 2018). From our numerical simulations initialized with orbital elements from the transit fit, in the stable simulations in which no planetary orbits cross, we find that planets K2-266 d and K2-266 e are in true resonance for $8.1 \%$ of the time, as characterized by a librating resonance angle. This significant fraction makes K2-266 another member of the short list of stars hosting systems containing potentially resonant exoplanets. Additional transits, which will improve orbital period precision, would enable future refinement of this resonance fraction.

From the time-evolution of the MEGNO indicator, we find that the average draw from the posterior becomes noticeably chaotic after roughly 100 years. The high mutual inclination between validated planet K2-266 b and the rest of the planets is also intriguing. As shown in Figure 10, because of the high present-day inclination of $\mathrm{K} 2-266 \mathrm{~b}$, this system is rarely (perhaps $5 \%$ of the time) in a configuration where all six planets can be seen simultaneously from our current line of sight. A smaller number of planets are expected to be seen in transit most of the time. Similarly, it is possible that the system hosts more than six planets, but we are seeing only six of them in transit at the current epoch. Tighter limits on the planetary posteriors will allow for a more precise determination of the future transit probability for each (known) planet, and will place constraints on any additional bodies in the system. In future work, a numerical survey of the parameter space subtended by the measured posteriors might allow for additional constraints on planetary parameters based on dynamical stability limits.

The formation of misaligned orbits, such as that of K2-266 b, remains an open problem. Petrovich et al. (2018) proposes that most USP planets form through non-linear secular interactions ("secular chaos"). In this scenario, the proto-USP planet starts with an orbital period of 5-10 days, is excited to high eccentricity, and is subsequently tidally captured onto a shortperiod orbit. Note that the process that leads to high eccentricity (e.g., planet-planet scattering) could also produce inclined orbits. As a result, one potential signature of this process could be a high mutual inclination for the USP planet relative to the other planets, as seen in this system. However, most companions to a USP planet generated in this manner would generally have orbital periods of 10 days or larger, whereas the K2-266 system has two planets with shorter periods of only six and seven days. For this secular chaos mechanism to form this system, the six- and seven-day planets would need to migrate inward after the eccentricity excitement and subsequent circularization of the USP planet; yet, as K2-266 b may have an unevaporated atmosphere based on current radius estimates, a dynamical history allowing it to form further from the star (subject to less photoevaporation) seems favorable. 
Table 7

Median Values and 68\% Confidence Intervals for Planetary Parameters of EPIC 248435395 from EXOFASTv2

\begin{tabular}{|c|c|c|}
\hline \multicolumn{2}{|c|}{ Planetary Parameters: } & \multirow{2}{*}{$\begin{array}{l}\text { EPIC } 248435395.01 \\
8.53467_{-0.00063}^{+0.00073}\end{array}$} \\
\hline$P$ & Period (days) & \\
\hline$R_{P}$ & Radius $\left(R_{\oplus}\right)$ & $10.6_{-8.5}^{+2.7}$ \\
\hline$T_{C}$ & Time of conjunction $\left(\mathrm{BJD}_{\mathrm{TDB}}\right)$ & $2457907.9884_{-0.0039}^{+0.0034}$ \\
\hline$T_{0}$ & $\begin{array}{l}\text { Optimal conjunction } \\
\text { time }\left(\mathrm{BJD}_{\mathrm{TDB}}\right)\end{array}$ & $2457925.0578_{-0.0028}^{+0.0025}$ \\
\hline$a$ & Semimajor axis (au) & $0.0684 \pm 0.0012$ \\
\hline$i$ & Inclination (degrees) & $87.22_{-0.23}^{+0.30}$ \\
\hline$T_{\text {eq }}$ & Equilibrium temperature $(\mathrm{K})$ & $530.3_{-7.7}^{+7.6}$ \\
\hline$R_{P} / R_{*}$ & Radius of planet in stellar radii & $0.147_{-0.12}^{+0.042}$ \\
\hline$a / R_{*}$ & Semimajor axis in stellar radii & $22.7_{-1.4}^{+1.3}$ \\
\hline$\delta$ & Transit depth (fraction) & $0.022_{-0.021}^{+0.014}$ \\
\hline Depth & Flux decrement at mid-transit & $0.00096_{-0.00026}^{+0.00020}$ \\
\hline$\tau$ & $\begin{array}{l}\text { Ingress/egress transit dura- } \\
\text { tion (days) }\end{array}$ & $0.0154 \pm 0.0016$ \\
\hline$T_{14}$ & Total transit duration (days) & $0.0317_{-0.0026}^{+0.0061}$ \\
\hline$T_{\text {FWHM }}$ & FWHM transit duration (days) & $0.0158_{-0.0013}^{+0.0032}$ \\
\hline$b$ & Transit impact parameter & $1.119_{-0.14}^{+0.043}$ \\
\hline$\delta_{S, 3.6 \mu \mathrm{m}}$ & $\begin{array}{l}\text { Blackbody eclipse depth at } \\
3.6 \mu \mathrm{m}(\mathrm{ppm})\end{array}$ & $21_{-20}^{+15}$ \\
\hline$\delta_{S, 4.5 \mu \mathrm{m}}$ & $\begin{array}{l}\text { Blackbody eclipse depth at } \\
\quad 4.5 \mu \mathrm{m}(\mathrm{ppm})\end{array}$ & $74_{-71}^{+49}$ \\
\hline$\langle F\rangle$ & Incident flux $\left(10^{9} \mathrm{erg} \mathrm{s}^{-1} \mathrm{~cm}^{-2}\right)$ & $0.0179 \pm 0.0010$ \\
\hline$T_{P}$ & Time of periastron $\left(\mathrm{BJD}_{\mathrm{TDB}}\right)$ & $2457907.9884_{-0.0039}^{+0.0034}$ \\
\hline$T_{S}$ & Time of eclipse (BJD $\left.\mathrm{TDB}_{\mathrm{TDB}}\right)$ & $2457912.2558_{-0.0036}^{+0.0031}$ \\
\hline$T_{A}$ & Time of ascending node $\left(\mathrm{BJD}_{\mathrm{TDB}}\right)$ & $2457905.8548_{-0.0040}^{+0.0035}$ \\
\hline$T_{D}$ & $\begin{array}{l}\text { Time of descending } \\
\text { node }\left(\mathrm{BJD}_{\mathrm{TDB}}\right)\end{array}$ & $2457910.1221_{-0.0037}^{+0.0032}$ \\
\hline$d / R_{*}$ & Separation at mid-transit & $22.7_{-1.4}^{+1.3}$ \\
\hline$P_{T}$ & A priori non-grazing transit prob & $0.0387_{-0.0039}^{+0.0043}$ \\
\hline$P_{T, G}$ & A priori transit prob & $0.0499_{-0.0045}^{+0.0039}$ \\
\hline \multicolumn{2}{|c|}{ Wavelength Parameters: } & Kepler \\
\hline$u_{1}$ & Linear limb-darkening coeff & $0.35_{-0.14}^{+0.16}$ \\
\hline$u_{2}$ & Quadratic limb-darkening coeff & $0.37_{-0.14}^{+0.11}$ \\
\hline \multicolumn{2}{|c|}{ Transit Parameters: } & dat UT p248-43-53 (Kepler) \\
\hline$\sigma^{2}$ & Added Variance & $0.000000000090_{-0.000000000054}^{+0.0000000057}$ \\
\hline$F_{0}$ & Baseline flux & $1.0000000 \pm 0.0000026$ \\
\hline
\end{tabular}

\section{Conclusion}

We present the discovery of up to six planets transiting the bright ( $K=8.9$ ) nearby ( $~ 78$ pc) star K2-266 (EPIC 248435473). From a global model where we simultaneously fit all six planetary signals, we find that the six planets have periods between 0.66 to 56.7 days, and radii of 0.65 to $3.3 R_{\oplus}$. From analyzing transit timing variations, we are able to confirm the two warm Neptunes ( $\mathrm{d}$ and e), constraining their masses to be $m_{d}=8.9_{-3.8}^{+5.7} M_{\oplus}$ and $m_{e}=14.3_{-5.0}^{+6.4} M_{\oplus}$. Additionally, we are able to validate the planetary nature of planets $\mathrm{b}$ and $\mathrm{c}$. Future follow-up observations should aim to confirm the transits of K2-266.02 and .06 through high photometric precision observations with facilities like NASA's Spitzer Space Telescope. Our analysis shows that the inner USP planet, K2-266 b, has an inclination of $75^{\circ} .3$, while the other five planets are consistent with an inclination between $87^{\circ}$ and $90^{\circ}$. This corresponds to a mutual misalignment of $>12.5$ which may indicate that planet $b$ did not form in the same way as the others. The brightness of K2-266 and the relatively large sizes of the sub-Neptune planets $\mathrm{d}$ and e make them great targets for atmospheric characterization observation with current facilities like $H S T$ and future facilities like JWST.

We thank Chelsea Huang, Laura Kreidberg, George Zhou, and Li Zeng for their valuable conversations. Work performed by J.E.R. was supported by the Harvard Future Faculty Leaders Postdoctoral fellowship. A.V.'s contribution to this work was performed under contract with the California Institute of Technology (Caltech)/Jet Propulsion Laboratory (JPL) funded by NASA through the Sagan Fellowship Program executed by the NASA Exoplanet Science Institute.

This work has made use of data from the European Space Agency (ESA) mission Gaia (https://www.cosmos.esa.int/ gaia), processed by the Gaia Data Processing and Analysis Consortium (DPAC,https://www.cosmos.esa.int/web/gaia/ dpac/consortium). Funding for the DPAC has been provided by national institutions, particularly the institutions participating in the Gaia Multilateral Agreement.

This paper includes data collected by the $K 2$ mission. Funding for the $K 2$ mission is provided by the NASA Science Mission directorate.

A portion of this work was supported by a NASA Keck PI Data Award, administered by the NASA Exoplanet Science Institute. Data presented herein were obtained at the W. M. Keck Observatory from telescope time allocated to the National Aeronautics and Space Administration through the agency's scientific partnership with the California Institute of Technology and the University of California. The Observatory was made possible by the generous financial support of the W. M. Keck Foundation.

The authors wish to recognize and acknowledge the very significant cultural role and reverence that the summit of Maunakea has always had within the indigenous Hawaiian community. We are most fortunate to have the opportunity to conduct observations from this mountain.

\section{ORCID iDs}

Joseph E. Rodriguez (10 https://orcid.org/0000-0001-8812-0565 Juliette C. Becker (1) https://orcid.org/0000-0002-7733-4522 Jason D. Eastman (1) https://orcid.org/0000-0003-3773-5142 Andrew Vanderburg (1) https://orcid.org/0000-0001-7246-5438 Tali Khain () https://orcid.org/0000-0001-7721-6457

Samuel N. Quinn (1) https://orcid.org/0000-0002-8964-8377 Courtney D. Dressing (1) https://orcid.org/0000-0001-8189-0233 Joshua E. Schlieder (10 https://orcid.org/0000-0001-5347-7062 David W. Latham (i) https://orcid.org/0000-0001-9911-7388 Saul Rappaport (1) https://orcid.org/0000-0003-3182-5569 Fred C. Adams 나 https://orcid.org/0000-0002-8167-1767 Allyson Bieryla (1) https://orcid.org/0000-0001-6637-5401 Michael L. Calkins (10 https://orcid.org/0000-0002-2830-5661 Keivan G. Stassun ำ https://orcid.org/0000-0002-3481-9052

\section{References}

Adams, F. C. 2011, ApJ, 730, 27

Adams, F. C., \& Laughlin, G. 2006, ApJ, 649, 1004

Agol, E., Steffen, J., Sari, R., \& Clarkson, W. 2005, MNRAS, 359, 567 Akeson, R. L., Chen, X., Ciardi, D., et al. 2013, PASP, 125, 989

Alencar, S. H. P., Teixeira, P. S., Guimarães, M. M., et al. 2010, A\&A, 519, A88

ALMA Partnership, Brogan, C. L., Pérez, L. M., et al. 2015, ApJL, 808, L3 Ansdell, M., Gaidos, E., Williams, J. P., et al. 2016a, MNRAS, 462, L101 Ansdell, M., Gaidos, E., Rappaport, S. A., et al. 2016b, ApJ, 816, 69 Ballard, S., \& Johnson, J. A. 2016, ApJ, 816, 66 
Barclay, T., Rowe, J. F., Lissauer, J. J., et al. 2013, Natur, 494, 452 Batalha, N. M., Borucki, W. J., Bryson, S. T., et al. 2011, ApJ, 729, 27 Becker, J. C., \& Adams, F. C. 2016, MNRAS, 455, 2980 Becker, J. C., \& Adams, F. C. 2017, MNRAS, 468, 549 Beichman, C., Benneke, B., Knutson, H., et al. 2014, PASP, 126, 1134 Bouvier, J., Chelli, A., Allain, S., et al. 1999, A\&A, 349, 619 Brakensiek, J., \& Ragozzine, D. 2016, ApJ, 821, 47 Buchhave, L. A., Bakos, G. Á., Hartman, J. D., et al. 2010, ApJ, 720, 1118 Buchhave, L. A., Bizzarro, M., Latham, D. W., et al. 2014, Natur, 509, 593 Carter, J. A., Agol, E., Chaplin, W. J., et al. 2012, Sci, 337, 556 Chambers, J. E. 1999, MNRAS, 304, 793

Charbonneau, D., Berta, Z. K., Irwin, J., et al. 2009, Natur, 462, 891 Chen, J., \& Kipping, D. 2017, ApJ, 834, 17

Choi, J., Dotter, A., Conroy, C., et al. 2016, ApJ, 823, 102

Christiansen, J. L., Vanderburg, A., Burt, J., et al. 2017, AJ, 154, 122

Ciardi, D. R., Crossfield, I. J. M., Feinstein, A. D., et al. 2018, AJ, 155, 10

Cincotta, P. M., Giordano, C. M., \& Simó, C. 2003, PhyD, 182, 151

Cody, A. M., Stauffer, J., Baglin, A., et al. 2014, AJ, 147, 82

Crossfield, I. J. M., \& Kreidberg, L. 2017, AJ, 154, 261

Crossfield, I. J. M., Ciardi, D. R., Isaacson, H., et al. 2017, AJ, 153, 255

Cutri, R. M., et al. 2014, yCat, 2328, 0

Cutri, R. M., Skrutskie, M. F., van Dyk, S., et al. 2003, yCat, 2246, 0

D’Antona, F., \& Mazzitelli, I. 1994, ApJS, 90, 467

David, T. J., Crossfield, I. J. M., Benneke, B., et al. 2018, AJ, 155, 222

Dawson, R. I., \& Fabrycky, D. C. 2010, ApJ, 722, 937

Deck, K. M., \& Agol, E. 2015, ApJ, 802, 116

Deck, K. M., Agol, E., Holman, M. J., \& Nesvorný, D. 2014, ApJ, 787, 132

Deck, K. M., Holman, M. J., Agol, E., et al. 2012, ApJL, 755, L21

Denham, P., Naoz, S., Hoang, B.-M., Stephan, A. P., \& Farr, W. M. 2018, arXiv: 1802.00447

Diez Alonso, E., Suarez Gomez, S. L., Gonzalez Hernandez, J. I., et al. 2018, arXiv: 1801.06249

Dotter, A. 2016, ApJS, 222, 8

Dragomir, D., Matthews, J. M., Eastman, J. D., et al. 2013, ApJL, 772, L2

Dressing, C. D., Newton, E. R., Schlieder, J. E., et al. 2017, ApJ, 836, 167

Eastman, J. 2017, EXOFASTv2: Generalized Publication-quality Exoplanet Modeling Code, Astrophysics Source Code Library, ascl:1710.003

Eastman, J., Gaudi, B. S., \& Agol, E. 2013, PASP, 125, 83

Fabrycky, D., \& Tremaine, S. 2007, ApJ, 669, 1298

Fabrycky, D. C., Lissauer, J. J., Ragozzine, D., et al. 2014, ApJ, 790, 146

Fang, J., \& Margot, J.-L. 2012, ApJ, 761, 92

Fúrész, G. 2008, PhD thesis, Univ. Szeged

Figueira, P., Marmier, M., Boué, G., et al. 2012, A\&A, 541, A139

Fischer, D. A., Marcy, G. W., Butler, R. P., et al. 2008, ApJ, 675, 790

Flewelling, H. A., Magnier, E. A., Chambers, K. C., et al. 2016, arXiv:1612. 05243

Foreman-Mackey, D., Hogg, D. W., Lang, D., \& Goodman, J. 2013, PASP, 125,306

Fressin, F., Torres, G., Charbonneau, D., et al. 2013, ApJ, 766, 81

Fulton, B. J., Petigura, E. A., Howard, A. W., et al. 2017, AJ, 154, 109

Gaia Collaboration, Brown, A. G. A., Vallenari, A., et al. 2018, arXiv:1804. 09365

Gaia Collaboration, Brown, A. G. A., Vallenari, A., et al. 2016, A\&A, 595, A2 Gillon, M., Triaud, A. H. M. J., Demory, B.-O., et al. 2017, Natur, 542, 456

Goodman, J., \& Weare, J. 2010, Communications in Applied Mathematics and Computational Science, 5, 65

Hadden, S., \& Lithwick, Y. 2014, ApJ, 787, 80

Hadden, S., \& Lithwick, Y. 2016, ApJ, 828, 44

Hadden, S., \& Lithwick, Y. 2017, AJ, 154, 5

Hansen, B. M. S. 2017, MNRAS, 467, 1531

Hansen, B. M. S., \& Murray, N. 2013, ApJ, 775, 53

Heap, S. R., Lindler, D. J., Lanz, T. M., et al. 2000, ApJ, 539, 435

Henden, A. A., Templeton, M., Terrell, D., et al. 2016, yCat, 2336, 0

Herter, T. L., Henderson, C. P., Wilson, J. C., et al. 2008, Proc. SPIE, 7014, $70140 \mathrm{X}$

Hirano, T., Fukui, A., Mann, A. W., et al. 2016, ApJ, 820, 41

Hirano, T., Dai, F., Livingston, J. H., et al. 2018, AJ, 155, 124

Holczer, T., Mazeh, T., Nachmani, G., et al. 2016, ApJS, 225, 9

Holman, M. J., \& Murray, N. W. 2005, Sci, 307, 1288

Howard, A. W., Marcy, G. W., Bryson, S. T., et al. 2012, ApJS, 201, 15

Howell, S. B., Sobeck, C., Haas, M., et al. 2014, PASP, 126, 398

Huber, D., Bryson, S. T., Haas, M. R., et al. 2016, ApJS, 224, 2

Jensen, E. L. N., \& Akeson, R. 2014, Natur, 511, 567

Jontof-Hutter, D., Weaver, B. P., Ford, E. B., Lissauer, J. J., \& Fabrycky, D. C. 2017, AJ, 153, 227

Jontof-Hutter, D., Ford, E. B., Rowe, J. F., et al. 2016, ApJ, 820, 39
Kalirai, J. 2018, ConPh, 59, 251

Ketchum, J. A., Adams, F. C., \& Bloch, A. M. 2013, ApJ, 762, 71

Kipping, D. M., Huang, X., Nesvorný, D., et al. 2015, ApJL, 799, L14

Lissauer, J. J., Ragozzine, D., Fabrycky, D. C., et al. 2011, ApJS, 197, 8

Lissauer, J. J., Marcy, G. W., Rowe, J. F., et al. 2012, ApJ, 750, 112

Livingston, J. H., Dai, F., Hirano, T., et al. 2018, AJ, 155, 115

Loomis, R. A., Öberg, K. I., Andrews, S. M., \& MacGregor, M. A. 2017, ApJ, 840,23

Lopez, E. D. 2017, MNRAS, 472, 245

Luger, R., Sestovic, M., Kruse, E., et al. 2017, NatAs, 1, 0129

Mann, A. W., Feiden, G. A., Gaidos, E., Boyajian, T., \& von Braun, K. 2015, ApJ, 804, 64

Mann, A. W., Gaidos, E., \& Ansdell, M. 2013a, ApJ, 779, 188

Mann, A. W., Gaidos, E., Kraus, A., \& Hilton, E. J. 2013b, ApJ, 770, 43

Mann, A. W., Vanderburg, A., Rizzuto, A. C., et al. 2018, AJ, 155, 4

Mayo, A. W., Vanderburg, A., Latham, D. W., et al. 2018, AJ, 155, 136

Millholland, S., Laughlin, G., Teske, J., et al. 2018, AJ, 155, 106

Mills, S. M., \& Fabrycky, D. C. 2017, AJ, 153, 45

Mills, S. M., Fabrycky, D. C., Migaszewski, C., et al. 2016, Natur, 533, 509

Morales-Calderón, M., Stauffer, J. R., Hillenbrand, L. A., et al. 2011, ApJ, 733,50

Morton, T. D. 2012, ApJ, 761, 6

Morton, T. D. 2015, VESPA: False Positive Probabilities Calculator, Astrophysics Source Code Library, ascl:1503.011

Morton, T. D., \& Winn, J. N. 2014, ApJ, 796, 47

Muirhead, P. S., Becker, J., Feiden, G. A., et al. 2014, ApJS, 213, 5

Mullally, F., Thompson, S. E., Coughlin, J. L., Burke, C. J., \& Rowe, J. F. 2018, AJ, 155, 210

Mustill, A. J., Davies, M. B., \& Johansen, A. 2017, MNRAS, 468, 3000

Nagasawa, M., \& Ida, S. 2011, ApJ, 742, 72

Nesvorný, D., \& Vokrouhlický, D. 2014, ApJ, 790, 58

Newton, E. R., Charbonneau, D., Irwin, J., et al. 2014, AJ, 147, 20

Newton, E. R., Charbonneau, D., Irwin, J., \& Mann, A. W. 2015, ApJ, 800, 85

Niraula, P., Redfield, S., Dai, F., et al. 2017, AJ, 154, 266

Owen, J. E., \& Wu, Y. 2013, ApJ, 775, 105

Owen, J. E., \& Wu, Y. 2017, ApJ, 847, 29

Palla, F., \& Stahler, S. W. 1991, ApJ, 375, 288

Paxton, B., Bildsten, L., Dotter, A., et al. 2011, ApJS, 192, 3

Paxton, B., Cantiello, M., Arras, P., et al. 2013, ApJS, 208, 4

Paxton, B., Marchant, P., Schwab, J., et al. 2015, ApJS, 220, 15

Petrovich, C., Deibert, E., \& Wu, Y. 2018, arXiv:1804.05065

Press, W. H., Teukolsky, S. A., Vetterling, W. T., \& Flannery, B. P. 1992, Numerical Recipes in C. The Art of Scientific Computing (Cambridge: Cambridge Univ. Press)

Rappaport, S., Vanderburg, A., Nelson, L., et al. 2017, MNRAS, 471, 948

Rasio, F. A., \& Ford, E. B. 1996, Sci, 274, 954

Rein, H., \& Liu, S.-F. 2012, A\&A, 537, A128

Rein, H., \& Tamayo, D. 2015, MNRAS, 452, 376

Ricker, G. R., Winn, J. N., Vanderspek, R., et al. 2015, JATIS, 1, 014003

Rivera, E. J., Laughlin, G., Butler, R. P., et al. 2010, ApJ, 719, 890

Rodriguez, J. E., Vanderburg, A., Eastman, J. D., et al. 2018, AJ, 155, 72

Rodriguez, J. E., Zhou, G., Vanderburg, A., et al. 2017, AJ, 153, 256

Sanchis-Ojeda, R., Rappaport, S., Winn, J. N., et al. 2014, ApJ, 787, 47

Schlegel, D. J., Finkbeiner, D. P., \& Davis, M. 1998, ApJ, 500, 525

Shallue, C. J., \& Vanderburg, A. 2018, AJ, 155, 94

Skrutskie, M. F., Cutri, R. M., Stiening, R., et al. 2006, AJ, 131, 1163

Spalding, C., Batygin, K., \& Adams, F. C. 2014, ApJL, 797, L29

Stassun, K. G., \& Torres, G. 2018, ApJ, 862, 61

Steffen, J. H., Fabrycky, D. C., Agol, E., et al. 2013, MNRAS, 428, 1077

Swift, J. J., Johnson, J. A., Morton, T. D., et al. 2013, ApJ, 764, 105

Vacca, W. D., Cushing, M. C., \& Rayner, J. T. 2003, PASP, 115, 389

Van Eylen, V., \& Albrecht, S. 2015, ApJ, 808, 126

Van Eylen, V., Lund, M. N., Silva Aguirre, V., et al. 2014, ApJ, 782, 14

Van Laerhoven, C., \& Greenberg, R. 2012, CeMDA, 113, 215

Vanderburg, A., \& Johnson, J. A. 2014, PASP, 126, 948

Vanderburg, A., Latham, D. W., Buchhave, L. A., et al. 2016a, ApJS, 222, 14

Vanderburg, A., Bieryla, A., Duev, D. A., et al. 2016b, ApJL, 829, L9

Weiss, L. M., \& Marcy, G. W. 2014, ApJL, 783, L6

Winn, J. N., Sanchis-Ojeda, R., Rogers, L., et al. 2017, AJ, 154, 60

Wu, Y., \& Lithwick, Y. 2011, ApJ, 735, 109

Wu, Y., \& Murray, N. 2003, ApJ, 589, 605

Xie, J.-W., Dong, S., Zhu, Z., et al. 2016, PNAS, 113, 11431

Zacharias, N., Finch, C., \& Frouard, J. 2017, yCat, 1340, 0

Zeng, L., Sasselov, D. D., \& Jacobsen, S. B. 2016, ApJ, 819, 127

Zhu, W., Petrovich, C., Wu, Y., Dong, S., \& Xie, J. 2018, arXiv:1802.09526 